\title{
Tykhonov well-posedness of split problems
}

Qiao-yuan Shu', Mircea Sofonea ${ }^{2,3}$ and Yi-bin Xiao ${ }^{2^{*}}$

\section{"Correspondence:}

xiaoyb9999@hotmail.com

${ }^{2}$ School of Mathematical Sciences, University of Electronic Science and Technology of China, Chengdu, P.R. China

Full list of author information is available at the end of the article

\section{Springer}

\begin{abstract}
In (J. Optim. Theory Appl. 183:139-157, 2019) we introduced and studied the concept of well-posedness in the sense of Tykhonov for abstract problems formulated on metric spaces. Our aim of this current paper is to extend the results in (J. Optim. Theory Appl. 183:139-157, 2019) to a system which consists of two independent problems denoted by $P$ and $Q$, coupled by a nonlinear equation. Following the terminology used in literature we refer to such a system as a split problem. We introduce the concept of well-posedness for the abstract split problem and provide its characterization in terms of metric properties for a family of approximating sets and in terms of the well-posedness for the problems $P$ and $Q$, as well. Then we illustrate the applicability of our results in the study of three relevant particular cases: a split variational-hemivariational inequality, an elliptic variational inequality and a history-dependent variational inequality. We describe each split problem and clearly indicate the family of approximating sets. We provide necessary and sufficient conditions which guarantee the well-posedness of the split variational-hemivariational inequality. Moreover, under appropriate assumptions on the data, we prove the well-posedness of the split elliptic variational inequality as well as the well-posedness of the split history-dependent variational inequality. We illustrate our abstract results with various examples, part of them arising in contact mechanics.
\end{abstract}

Keywords: Split problem; Tykhonov well-posedness; Variational inequality; Hemivariational inequality; History-dependent operator; Contact problem

\section{Introduction}

Variational inequalities and their related problems represent a powerful mathematical tool used in the study of various nonlinear boundary value problems. They have important applications in mechanics, physics, economics and engineering sciences. The theory of variational inequalities was developed in the early 1960s, by using arguments of monotonicity and convexity. An unavoidable reference in the field is the book of Kinderlehrer and Stampacchia[16], together with its second edition [17]. The literature on variational inequalities and their related problems also include the references $[1,9,10,25,41,42]$ and, more recently, [34]. The concept of split variational inequality was introduced in [6]. It concerns a couple of variational inequalities whose solutions, denoted $u$ and $\sigma$, are constrained to satisfy an equality of the form $\sigma=T u$. Split variational inequalities have many applications in practical problems arising from signal recovery, image processing and radiation therapy. References in the field are $[5,12,23]$.

(c) The Author(s) 2020. This article is licensed under a Creative Commons Attribution 4.0 International License, which permits use sharing, adaptation, distribution and reproduction in any medium or format, as long as you give appropriate credit to the original author(s) and the source, provide a link to the Creative Commons licence, and indicate if changes were made. The images or other third party material in this article are included in the article's Creative Commons licence, unless indicated otherwise in a credit line to the material. If material is not included in the article's Creative Commons licence and your intended use is not permitted by statutory regulation or exceeds the permitted use, you will need to obtain permission directly from the copyright holder. To view a copy of this licence, visit http://creativecommons.org/licenses/by/4.0/. 
Hemivariational inequalities have been introduced in order to solve nonsmooth and nonmontone problems arising in mechanics and, in particular, in solid and contact mechanics. The theory of hemivariational inequalities started in the early 1980s and has as main ingredients the properties of the subdifferential in the sense of Clarke, defined for locally Lipschitz functions [2, 21, 24, 25, 31, 38, 39]. The concept of split hemivariational inequality was inspired by the concept of split variational inequality and was studied in a number of recent papers, including [27]. There, a split hemivariational inequality was defined by a couple of hemivariational inequalities whose solutions satisfy an additional equation of the form $\sigma=T u$.

Variational-hemivariational inequalities represent a special class of inequalities, in which both convex and nonconvex functions are involved [26,30]. There, various existence and uniqueness results are presented in the abstract framework of reflexive Banach spaces and used in a study of mathematical models arising in contact mechanics. Split variational-hemivariational inequalities have been considered in [15]. There, equivalence results which characterize the well-posedness of such inequalities were obtained.

History-dependent operators represent a special class of nonlinear operators defined on the space of continuous functions. They arise in nonlinear analysis, solid mechanics and contact mechanics. In contact mechanics, history-dependent operators are used in order to model various memory effects which could appear in the constitutive laws of materials and in the statement of contact conditions and friction laws. Inequality problems with history-dependent operators have been studied intensively in the last decade, in the variational, the hemivariational and the variational-hemivariational cases [21, 29, 30, 35].

Tykhonov's well-posedness concept was introduced in [36] for an unconstrained minimization problem and then extended in the study of various mathematical problems including variational and hemivariational inequalities, inclusions, fixed point and saddle point problems. It is based on two main ingredients: the existence of a unique solution for the problem and the convergence to it of any approximating sequence. The notion of approximating sequence varies from problem to problem but, in general, is constructed based on a family of approximating sets $\{\Omega(\varepsilon)\}_{\varepsilon>0}$ where $\varepsilon$ is a small parameter which converges to zero. The literature in the field is extensive. For instance, the well-posedness of variational inequalities was first studied in $[19,20]$. It was extended to hemivariational inequalities in $[3,11,13,14,27,33,37,40]$. In [37] the authors deal with well-posedness of a hemivariational inequality and its corresponding inclusion problem and provide a metric characterization of well-posedness for such problem, together with various equivalence results. The papers [27] and [15] extend these results in the study of split hemivariational and split variational-hemivariational inequalities, respectively.

In our recent paper [32] we introduced the concept of well-posedness in the sense of Tykhonov (well-posedness, for short) for a class of abstract problems in metric spaces. We obtained metric characterizations of the well-posedness of such kind of problems, then we illustrated the use of these abstract results in the study of several types of problems, including history-dependent variational and hemivariational inequalities. Our results in [32] provide a general framework in which the well-posedness of a large number of problems already studied in the literature cast.

The present paper represents a natural continuation of [32], since our aim is to extend the results in [32] to abstract split problems. Here and below, by a split problem we understand a mathematical object $\mathcal{M}$, composed of two problems $P$ and $Q$ with solutions $u$ 
and $\sigma$, respectively, associated to an implicit equation of the form $G(u, \sigma)=f$. For such a problem we use the notation $\mathcal{M}=\mathcal{M}(P, Q, G, f)$. We use in a constructive way the framework introduced in [32] and provide necessary and sufficient conditions which guarantee the well-posedness of the split problem $\mathcal{M}$, expressed either in terms of metric characterization of a family of approximating sets or in terms of the well-posedness of the problems $P$ and $Q$. This represents the first trait of novelty of our paper. Next, we study the wellposedness of three representative split problems: a variational-hemivariational inequality, an elliptic variational inequality and a history-dependent variational inequality. The study is carried out by using our abstract theory and leads to new and nonstandard results with potential applications in mechanics. For instance, it could be used to obtain convergence results which establish the link between different models of contact. This represents the second trait of novelty of our current paper.

The rest of the paper is structured as follows. In Sect. 2 we introduce the functional framework of split problems, then we state and prove three general results, Theorems 10, 13 and 14. Section 3 is devoted to results on the split variational-hemivariational inequality. Here we extend and complete our previous results obtained in [27]. We illustrate the abstract results in Sects. 3 and 4 with some elementary examples. In Sect. 4 we consider a split variational inequality for which we prove a well-posedness result. Finally, in Sect. 5, we state and prove the well-posedness for a split history-dependent variational inequality. We illustrate the abstract results in Sects. 4 and 5 with two examples which arise in contact mechanics.

We end this Introduction with the remark that the split problems we consider below in this paper are denoted by $\mathcal{M}$ or $\mathcal{M}(P, Q, G, f)$, even if their statement vary from section to section and from example to example. Unless stated otherwise, the references to a split problem $\mathcal{M}$ concern the problem $\mathcal{M}$ introduced in the corresponding section or example.

\section{Problem statement and abstract results}

Everywhere in this paper $X, Y, Z$ are assumed to be normed spaces, unless stated otherwise. The elements of $X$ will be denoted by $u, v, \ldots$ and the elements of $Y$ by $\sigma, \tau, \ldots$. The norms on these spaces will be denoted by $\|\cdot\|_{X},\|\cdot\|_{Y}$ and $\|\cdot\|_{Z}$, respectively. We also use the notation $\mathcal{X}$ for the product of the spaces $X$ and $Y$, i.e., $\mathcal{X}=X \times Y$. The elements of $\mathcal{X}$ will be denoted by $x=(u, \sigma), y=(v, \tau), \ldots$. We endow $\mathcal{X}$ with the norm $\|\cdot\|_{\mathcal{X}}$ given by

$$
\|x\|_{\mathcal{X}}=\|u\|_{X}+\|\sigma\|_{Y} \quad \forall x=(u, \sigma) \in \mathcal{X}
$$

We write $u_{n} \rightarrow u$ in $X$ for the strong convergence of a sequence $\left\{u_{n}\right\} \subset X$ to an element $u \in X$. The dual of $X$ will be denoted by $X^{*}$ and $\langle\cdot, \cdot\rangle_{X}$ will represent the duality paring mapping. We use similar notations for the spaces $Y, Z$ and $\mathcal{X}$. All the limits, upper limits and lower limits below are considered as $n \rightarrow \infty$, even if we do not specify it explicitly. For a sequence $\left\{\varepsilon_{n}\right\} \subset \mathbb{R}$ such that $\varepsilon_{n}>0$ for all $n \in \mathbb{N}$ and $\varepsilon_{n} \rightarrow 0$ as $n \rightarrow \infty$ we use the short hand notation $0<\varepsilon_{n} \rightarrow 0$.

We now recall some basic definitions and results introduced in [32]. Consider an abstract mathematical object $P$, called a generic "problem", defined on the normed space $X$. Problem $P$ could be an equation, an inclusion, a fixed point problem, an optimization problem or an inequality problem. We associate to $P$ a set $S_{P} \subset X$, called the "set of solutions". 
The elements of $S_{P}$ are called "solutions" of Problem $P$, i.e.,

$$
S_{p}=\{u \in X: u \text { is a solution to Problem } P\} .
$$

Note that the precise meaning of the term "solution" arises in the statement of each problem, as exemplified in [32]. We say that Problem $P$ has a unique solution if $S_{P}$ has a unique element, i.e., $S_{P}$ is a singleton. Finally, we associate to $P$ a family $\left\{\Omega^{P}(\varepsilon)\right\}_{\varepsilon>0}$ of subsets of $X$, called approximating sets, such that

$$
S_{P} \subset \Omega^{P}(\varepsilon) \quad \forall \varepsilon>0 .
$$

This family is assumed to be given and, again, its definition varies from example to example. With these notations, we recall the following definitions.

Definition 1 A sequence $\left\{u_{n}\right\} \subset X$ is called an approximating sequence for Problem $P$ if there exists a sequence $0<\varepsilon_{n} \rightarrow 0$ such that $u_{n} \in \Omega^{P}\left(\varepsilon_{n}\right)$ for each $n \in \mathbb{N}$.

Definition 2 Problem $P$ is said to be well-posed if it has a unique solution and every approximating sequence for $P$ converges in $X$ to the solution of $P$.

The well-posedness of Problem $P$ was characterized in the following result, proved in [32].

Theorem 3 Problem $P$ is well-posed if and only if its set of solution $S_{P}$ is nonempty and $\operatorname{diam}\left(\Omega^{P}(\varepsilon)\right) \rightarrow 0$ as $\varepsilon \rightarrow 0$.

Here and below, for a nonempty subset $A$ of any normed space $\left(W,\|\cdot\|_{W}\right)$ we use the notation $\operatorname{diam}(A)$ for the diameter of $A$, defined by equality

$$
\operatorname{diam}(A)=\sup _{a, b \in A}\|a-b\|_{W} .
$$

We also need the following definition considered in our previous paper [32].

Definition 4 A family $\left\{\Omega^{P}(\varepsilon)\right\}_{\varepsilon>0}$ of subsets of $X$ which satisfy (2.3) is called regular (for Problem $\mathrm{P}$ ) if the following hold:

(a) For all $\varepsilon_{1}, \varepsilon_{2}>0, \varepsilon_{1} \leq \varepsilon_{2} \Longrightarrow \Omega^{P}\left(\varepsilon_{1}\right) \subset \Omega^{P}\left(\varepsilon_{2}\right)$.

(b) Any convergent approximating sequence for $P$ converges to an element of $S_{P}$, i.e., if $\left\{u_{n}\right\}$ is an approximating sequence for $P$ and there exists $u \in X$ such that $u_{n} \rightarrow u$ in $X$ as $n \rightarrow \infty$, then $u \in S_{P}$.

After these preliminaries, we introduce the concept of split problem and its wellposedness. To this end, besides Problem $P$ endowed with its set of solutions $S_{P}$ and the associated family of approximating sets $\left\{\Omega^{P}(\varepsilon)\right\}_{\varepsilon>0}$ which satisfy condition (2.3), we consider a second problem, denoted by $Q$, defined on the space $Y$. We associate to Problem $Q$ a set of solutions $S_{Q} \subset Y$, as well as a family of subsets of $Y$, denoted by $\left\{\Omega^{Q}(\varepsilon)\right\}_{\varepsilon>0}$, such that

$$
S_{Q} \subset \Omega^{Q}(\varepsilon) \quad \forall \varepsilon>0
$$


Finally, we assume that $G: X \times Y \rightarrow Z$ is a given operator and $f$ is a given element of $Z$. With these data we construct a new problem which can be stated as follows.

Problem $\mathcal{M}$ Find an element $x=(u, \sigma) \in \mathcal{X}$ such that $u \in S_{P}, \sigma \in S_{Q}$ and $G(u, \sigma)=f$.

We say in what follows that $\mathcal{M}$ represents a split problem. Note that this new mathematical object is constructed by using the problems $P$ and $Q$, together with their sets of solutions $S_{P}$ and $S_{Q}$, the families of approximating sets $\left\{\Omega^{P}(\varepsilon)\right\}_{\varepsilon>0}$ and $\left\{\Omega^{Q}(\varepsilon)\right\}_{\varepsilon>0}$, the operator $G$ and the element $f$. For this reason, we should write

$$
\mathcal{M}=\mathcal{M}\left(P, Q, S_{P}, S_{Q},\left\{\Omega^{P}(\varepsilon)\right\}_{\varepsilon>0},\left\{\Omega^{Q}(\varepsilon)\right\}_{\varepsilon>0}, G, f\right) .
$$

Nevertheless, for simplicity, since the sets of solutions $S_{P}, S_{Q}$ are defined from the statement of the problems $P$ and $Q$, when the families of approximating sets $\left\{\Omega^{P}(\varepsilon)\right\}_{\varepsilon>0}$, $\left\{\Omega^{Q}(\varepsilon)\right\}_{\mathcal{E}>0}$ are fixed, we shall simply write

$$
\mathcal{M}=\mathcal{M}(P, Q, G, f)
$$

Finally, when no confusion arises, for a split problem $\mathcal{M}=\mathcal{M}(P, Q, G, f)$ we shall use the short hand notation $\mathcal{M}$.

Definition 5 An element $x=(u, \sigma) \in \mathcal{X}$ is said to be a solution to the split problem $\mathcal{M}=$ $\mathcal{M}(P, Q, G, f)$ if $u \in S_{P}, \sigma \in S_{Q}$ and $G(u, \sigma)=f$.

We denote in what follows by $S_{M}$ the set of solutions of the split problem $\mathcal{M}$, i.e.,

$$
S_{M}=\left\{x=(u, \sigma) \in \mathcal{X}: u \in S_{P}, \sigma \in S_{Q} \text { and } G(u, \sigma)=f\right\} .
$$

It follows from above that a split problem represents a systems of two independent problems $(P$ and $Q)$ coupled by a (usually nonlinear) equation $(G(u, \sigma)=f)$. Its solutions are elements $x=(u, \sigma)$ of the product space $\mathcal{X}=X \times Y$ such that their components solve the two problems $\left(u \in S_{P}\right.$ and $\left.\sigma \in S_{Q}\right)$ and, moreover, they satisfy an additional constraint $(G(u, \sigma)=f)$.

Note that this concept of split problem is quite general and includes as particular cases a number of split problems already studied in the literature, as mentioned in the Introduction. For instance, if both $P$ and $Q$ represent variational inequalities we say that $\mathcal{M}$ represents a split variational inequality, if $P$ and $Q$ are hemivariational inequalities we say that $\mathcal{M}$ represents a split hemivariational inequality and, if $P$ and $Q$ are inclusions we say that $\mathcal{M}$ represents a split inclusion. Nevertheless, we stress that the functional framework described above is very flexible and allows one to study a large number of split problems, in which $P$ and $Q$ could have different features. For instance, $P$ could be a variational inequality and $Q$ an inclusion, $P$ could be a fixed point problem and $Q$ an optimization problem, and so on. Also, we underline that, in contrast with the references mentioned above (where an explicit equation of the form $\sigma=T u$ was considered), the concept of split problem we consider here is more general, since it is based on an implicit equation of the form $G(u, \sigma)=f$. 
Finally, note that, in general, a split problem $\mathcal{M}=\mathcal{M}(P, Q, G, f)$ is over determined and has no solution. Indeed, assume that Problem $P$ has a unique solution $u_{0}$ and Problem $Q$ has a unique solution $\sigma_{0}$. Then, if the element $f \in Z$ is chosen such that $f=G\left(u_{0}, \sigma_{0}\right)$ it follows that the couple $x_{0}=\left(u_{0}, \sigma_{0}\right)$ is the unique solution to the split Problem $\mathcal{M}$. Nevertheless, if $f \neq G\left(u_{0}, \sigma_{0}\right)$, then Problem $\mathcal{M}$ has no solution. For this reason, it is important to identify classes of split problems $\mathcal{M}=\mathcal{M}(P, Q, G, f)$ which have solutions, without having a priori information on these solutions. And this is what we shall do in Sects. 4 and 5 of this paper, where we present two examples of well-posed split variational inequalities.

Next, to proceed our abstract construction, for each $\varepsilon>0$ we consider the set

$$
\Omega^{M}(\varepsilon)=\left\{x=(u, \sigma) \in \mathcal{X}: u \in \Omega^{P}(\varepsilon), \sigma \in \Omega^{Q}(\varepsilon),\|G(u, \sigma)-f\|_{Z} \leq \varepsilon\right\} .
$$

Note that $\Omega^{M}(\varepsilon) \subset \Omega^{P}(\varepsilon) \times \Omega^{Q}(\varepsilon)$. Moreover, (2.7), (2.3) and (2.5) imply that

$$
S_{M} \subset \Omega^{M}(\varepsilon) \quad \forall \varepsilon>0 .
$$

We now extend Definitions 1 and 2 to split problems.

Definition 6 A sequence $\left\{x_{n}\right\} \subset \mathcal{X}$ is called an approximating sequence for the split problem $\mathcal{M}=\mathcal{M}(P, Q, G, f)$ if there exists a sequence $0<\varepsilon_{n} \rightarrow 0$ such that $x_{n} \in \Omega^{M}\left(\varepsilon_{n}\right)$ for all $n \in \mathbb{N}$.

Definition 7 The split problem $\mathcal{M}$ is said to be well-posed if it has a unique solution and every approximating sequence for $\mathcal{M}$ converges in $\mathcal{X}$ to the solution of $\mathcal{M}$.

We now proceed with two elementary examples in the case when $X=Y=Z=\mathbb{R}$.

Example 8 Consider the split problem $\mathcal{M}=\mathcal{M}(P, Q, G, f)$ defined as follows.

$$
\left\{\begin{array}{c}
\text { Problem } P \text {. Find } u \in \mathbb{R} \text { such that } u^{3}-u^{2}+2 u-2=0 . \\
\Omega^{P}(\varepsilon)=\left\{u \in \mathbb{R}:\left|u^{3}-u^{2}+2 u-2\right| \leq \varepsilon\right\} \quad \forall \varepsilon>0 . \\
\text { Problem } Q . \text { Find } \sigma \in \mathbb{R} \text { such that } \sigma^{3}-2 \sigma^{2}+\sigma-2=0 . \\
\Omega^{Q}(\varepsilon)=\left\{\sigma \in \mathbb{R}:\left|\sigma^{3}-2 \sigma^{2}+\sigma-2\right| \leq \varepsilon\right\} \quad \forall \varepsilon>0 . \\
G(u, \sigma)=\sigma-u, \quad f \in \mathbb{R} .
\end{array}\right.
$$

It is easy to see that in this case $S_{P}=\{1\}$ and $S_{Q}=\{2\}$. Moreover, we have the following result.

Claim 1 The split problem $\mathcal{M}$ is well-posed if and only iff $=1$.

Proof Assume that $\mathcal{M}$ is well-posed. Then using Definitions 7 and 5 it follows that $G(1,2)=f$ and, therefore, $f=1$. Conversely, assume that $f=1$. Then, it is clear that the couple $x=(1,2)$ is the unique solution to Problem $\mathcal{M}$. Assume that $\left\{x_{n}\right\}$ is an approximating sequence for $\mathcal{M}$. We have $x_{n}=\left(u_{n}, \sigma_{n}\right)$ for each $n \in \mathbb{N}$ and, using Definition 6, (2.8) and (2.10), there exists $0<\varepsilon_{n} \rightarrow 0$ such that

$$
\left|u_{n}^{3}-u_{n}^{2}+2 u_{n}-2\right| \leq \varepsilon_{n}, \quad\left|\sigma_{n}^{3}-2 \sigma_{n}^{2}+\sigma_{n}-2\right| \leq \varepsilon_{n} \quad \forall n \in \mathbb{N} .
$$


Therefore,

$$
\left|u_{n}-1\right| \leq \frac{\varepsilon_{n}}{u_{n}^{2}+2} \leq \frac{\varepsilon_{n}}{2}, \quad\left|\sigma_{n}-2\right| \leq \frac{\varepsilon_{n}}{\sigma_{n}^{2}+1} \leq \varepsilon_{n} \quad \forall n \in \mathbb{N} .
$$

It follows from (2.11) that $u_{n} \rightarrow 1$ and $\sigma_{n} \rightarrow 2$, i.e., $x_{n} \rightarrow x$ in $\mathbb{R}^{2}$. We now use Definition 7 to see that the split problem $\mathcal{M}$ is well-posed, which concludes the proof of the claim.

Example 9 Consider the split problem $\mathcal{M}=\mathcal{M}(P, Q, G, f)$ in Example 8 in the case when $f=1$ and

$$
\begin{array}{ll}
\Omega^{P}(\varepsilon)=\left\{u \in \mathbb{R}:\left|u^{3}-u^{2}+2 u-2\right| \leq \varepsilon+2\right\} & \forall \varepsilon>0, \\
\Omega^{Q}(\varepsilon)=\left\{\sigma \in \mathbb{R}:\left|\sigma^{3}-2 \sigma^{2}+\sigma-2\right| \leq \varepsilon+2\right\} & \forall \varepsilon>0 .
\end{array}
$$

The unique solution of this problem is $x=(1,2)$. Nevertheless, it is easy to see that in this case the element $x^{\prime}=(0,1)$ belongs to the set $\Omega^{M}(\varepsilon)$, for each $\varepsilon>0$. Therefore, using Definition 6 it follows that the sequence $\left\{x_{n}^{\prime}\right\}$ defined by $x_{n}^{\prime}=x^{\prime}$ for each $n \in \mathbb{N}$ is an approximating sequence for Problem $\mathcal{M}$. Since this sequence does not converge to $x$, Definition 7 implies that the split problem $\mathcal{M}$ is not well-posed.

Our first result in this section is the following.

Theorem 10 The split problem $\mathcal{M}=\mathcal{M}(P, Q, G, f)$ is well-posed if and only if its solution set $S_{M}$ is nonempty and $\operatorname{diam}\left(\Omega^{M}(\varepsilon)\right) \rightarrow 0$ as $\varepsilon \rightarrow 0$.

Proof Assume that $\mathcal{M}$ is well-posed. Then, by definition, the set $S_{M}$ is a singleton and, therefore, $S_{M} \neq \emptyset$. Arguing by contradiction, we assume in what follows that $\operatorname{diam}\left(\Omega^{M}(\varepsilon)\right) \nrightarrow 0$ as $\varepsilon \rightarrow 0$. Then, there exist $\delta_{0} \geq 0$, a sequence $0<\varepsilon_{n} \rightarrow 0$ and two sequences $\left\{x_{n}\right\},\left\{y_{n}\right\} \subset \mathcal{X}$ such that $x_{n}, y_{n} \in \Omega^{M}\left(\varepsilon_{n}\right)$ and

$$
\left\|x_{n}-y_{n}\right\| \mathcal{X} \geq \frac{\delta_{0}}{2} \quad \forall n \in \mathbb{N}
$$

Now, since both $\left\{x_{n}\right\}$ and $\left\{y_{n}\right\} \subset \mathcal{X}$ are approximating sequences for Problem $\mathcal{M}$, the wellposedness of $\mathcal{M}$ implies that $x_{n} \rightarrow x$ and $y_{n} \rightarrow x$ in $\mathcal{X}$ where $x$ denotes the unique element of $S_{M}$. This is in contradiction with (2.12). We conclude from this that $\operatorname{diam}(\Omega(\varepsilon)) \rightarrow 0$ as $\varepsilon \rightarrow 0$.

Conversely, assume that $S_{M}$ is nonempty and $\operatorname{diam}\left(\Omega^{M}(\varepsilon)\right) \rightarrow 0$ as $\varepsilon \rightarrow 0$. We claim that $S_{M}$ is a singleton. Indeed, let $x, y \in S_{M}$. Then using (2.9) we deduce that $x, y \in \Omega^{M}(\varepsilon)$ for any $\varepsilon>0$. Thus,

$$
\|x-y\|_{\mathcal{X}} \leq \operatorname{diam}\left(\Omega\left(\varepsilon_{n}\right)\right) \rightarrow 0
$$

which implies that $x=y$ and proves the claim. We conclude from this that $\mathcal{M}$ has a unique solution, denoted in what follows by $x$. Let now $\left\{x_{n}\right\} \subset X$ be an approximating sequence for Problem $\mathcal{M}$. Then there exists a sequence $0<\varepsilon_{n} \rightarrow 0$ such that $x_{n} \in \Omega^{M}\left(\varepsilon_{n}\right)$ for each $n \in \mathbb{N}$. We use (2.9) to see that $x \in \Omega^{M}\left(\varepsilon_{n}\right)$ for each $n \in \mathbb{N}$ and, therefore,

$$
\left\|x-x_{n}\right\| \mathcal{X} \leq \operatorname{diam}\left(\Omega^{M}\left(\varepsilon_{n}\right)\right) \rightarrow 0
$$


This implies that $x_{n} \rightarrow x$ in $X$, which shows that Problem $\mathcal{M}$ is well-posed and concludes the proof.

Remark 11 Note that for the split problem $\mathcal{M}$ in Example 8 we have $\operatorname{diam}\left(\Omega^{P}(\varepsilon)\right) \rightarrow 0$ and $\operatorname{diam}\left(\Omega^{Q}(\varepsilon)\right) \rightarrow 0$ as $\varepsilon \rightarrow 0$, which imply that $\operatorname{diam}\left(\Omega^{M}(\varepsilon)\right) \rightarrow 0$ as $\varepsilon \rightarrow 0$. Therefore, Theorem 10 can be used in order to prove the Claim 1 in Example 8. Consider now the split problem $\mathcal{M}$ in Example 9. Note that in this case $\operatorname{diam}\left(\Omega^{M}(\varepsilon)\right) \geq\left\|x^{\prime}-x\right\|_{\mathbb{R}^{2}} \geq 2$ for each $\varepsilon>0$ and, therefore, Theorem 10 implies that this problem is not well-posed.

Note that Theorem 10 provides a necessary and sufficient condition for the wellposedness of Problem $\mathcal{M}$. Nevertheless, checking this condition requires one to prove that $S_{M} \neq \emptyset$, i.e., to prove the solvability of Problem $\mathcal{M}$. In what follows we introduce a second characterization for the well-posedness of Problem $\mathcal{M}$ in which condition $S_{M} \neq \emptyset$ is removed. To this end, we need the following definition.

Definition 12 The family $\left\{\Omega^{M}(\varepsilon)\right\}_{\varepsilon>0}$ of subsets in $\mathcal{X}$ given by (2.8) is said to be regular (for the split problem $\mathcal{M}$ ) if the families $\left\{\Omega^{P}(\varepsilon)\right\}$ and $\left\{\Omega^{Q}(\varepsilon)\right\}$ are regular for Problems $P$ and $Q$ in the sense of Definition 4. In other words, we say that the family $\left\{\Omega^{M}(\varepsilon)\right\}_{\varepsilon>0}$ is regular if the following hold:

(a) For all $\varepsilon_{1}, \varepsilon_{2}>0, \varepsilon_{1} \leq \varepsilon_{2} \Longrightarrow \Omega^{P}\left(\varepsilon_{1}\right) \subset \Omega^{P}\left(\varepsilon_{2}\right)$ and $\Omega^{Q}\left(\varepsilon_{1}\right) \subset \Omega^{Q}\left(\varepsilon_{2}\right)$.

(b) If $\left\{u_{n}\right\}$ is an approximating sequence of $P$ and there exists $u \in X$ such that $u_{n} \rightarrow u$ in $X$ as $n \rightarrow \infty$, then $u \in S_{P}$.

(c) If $\left\{\sigma_{n}\right\}$ is an approximating sequence of $Q$ and there exists $\sigma \in X$ such that $\sigma_{n} \rightarrow \sigma$ in $Y$ as $n \rightarrow \infty$, then $\sigma \in S_{Q}$.

Our second result in this section is the following.

Theorem 13 Assume that $X$ and $Y$ are Banach spaces, $\left\{\Omega^{M}(\varepsilon)\right\}_{\varepsilon>0}$ is a regular family of subsets in $\mathcal{X}$ defined by (2.8) and $G: X \times Y \rightarrow Z$ is a continuous operator. Then, the split problem $\mathcal{M}$ is well-posed if and only if for each $\varepsilon>0$ the set $\Omega^{M}(\varepsilon)$ is nonempty and $\operatorname{diam}\left(\Omega^{M}(\varepsilon)\right) \rightarrow 0$ as $\varepsilon \rightarrow 0$.

Proof Assume that $\mathcal{M}$ is well-posed. Then, we use Theorem 10 and inclusion (2.9) to see that for each $\varepsilon>0$ the set $\Omega^{M}(\varepsilon)$ is nonempty and, moreover, $\operatorname{diam}\left(\Omega^{M}(\varepsilon)\right) \rightarrow 0$ as $\varepsilon \rightarrow 0$.

Conversely, assume that for each $\varepsilon>0$ the set $\Omega^{M}(\varepsilon)$ is nonempty and, moreover, $\operatorname{diam}\left(\Omega^{M}(\varepsilon)\right) \rightarrow 0$ as $\varepsilon \rightarrow 0$. Let $\left\{x_{n}=\left(u_{n}, \sigma_{n}\right)\right\}$ be an approximating sequence for $\mathcal{M}$. Then there exists a sequence $0<\varepsilon_{n} \rightarrow 0$ such that $x_{n} \in \Omega^{M}\left(\varepsilon_{n}\right)$ for all $n \in \mathbb{N}$. This implies that

$$
u_{n} \in \Omega^{P}\left(\varepsilon_{n}\right), \sigma_{n} \in \Omega^{Q}\left(\varepsilon_{n}\right), \quad\left\|G\left(u_{n}, \sigma_{n}\right)-f\right\|_{Z} \leq \varepsilon_{n} \quad \forall n \in \mathbb{N} .
$$

Since $\operatorname{diam}\left(\Omega^{M}(\varepsilon)\right) \rightarrow 0$ as $\varepsilon>0$, for any $\delta>0$ there exists a positive integer $N_{\delta}$ such that

$$
\operatorname{diam}\left(\Omega^{M}\left(\varepsilon_{n}\right)\right) \leq \delta \quad \forall n \geq N_{\delta} .
$$

Let $n, m \in \mathbb{N}$ with $n, m \geq N_{\delta}$ and assume that $\varepsilon_{m} \leq \varepsilon_{n}$. Then, using condition (a) in Definition 12 and (2.13) we have

$$
u_{m} \in \Omega^{P}\left(\varepsilon_{n}\right), \sigma_{m} \in \Omega^{Q}\left(\varepsilon_{n}\right), \quad\left\|G\left(u_{m}, \sigma_{m}\right)-f\right\|_{Z} \leq \varepsilon_{n},
$$


which imply that $x_{m} \in \Omega^{M}\left(\varepsilon_{n}\right)$. Thus, since $x_{n}, x_{m} \in \Omega^{M}\left(\varepsilon_{n}\right)$, inequality (2.14) yields

$$
\left\|x_{m}-x_{n}\right\|_{X} \leq \delta
$$

This inequality holds even if $\varepsilon_{m}>\varepsilon_{n}$ since, in this case, $x_{n}, x_{m} \in \Omega^{M}\left(\varepsilon_{m}\right)$. We conclude from this that $\left\{x_{n}\right\}$ is a Cauchy sequence in $\mathcal{X}$ which is a Banach space since, as we recall, $X$ and $Y$ are assumed to be Banach spaces. It follows from this that there exists $x=(u, \sigma) \in \mathcal{X}$ such that

$$
x_{n} \rightarrow x \text { in } \mathcal{X}
$$

or, equivalently,

$$
u_{n} \rightarrow u \quad \text { in } X, \quad \sigma_{n} \rightarrow \sigma \quad \text { in } Y \text {. }
$$

The convergences (2.16) combined with conditions (b) and (c) in Definition 12 show that $u \in S_{P}$ and $\sigma \in S_{Q}$. Moreover, (2.13) and the continuity of the operator $G$ imply that $G(u, \sigma)=f$. It follows now from Definition 5 that the element $x=(u, \sigma)$ belongs to $S_{M}$ and, therefore, $S_{M} \neq \emptyset$. We are now in a position to use Theorem 10 to deduce that Problem $\mathcal{M}$ is well-posed, which concludes the proof.

The following result shows the link between the well-posedness of the split problem $\mathcal{M}$ (in the sense of Definition 6) and the well-posedness of its components $P$ and $Q$ (in the sense of Definition 2).

\section{Theorem 14}

(1) Assume that Problems $P$ and $Q$ are well-posed, denote by $u$ and $\sigma$ their solutions and assume that $G(u, \sigma)=f$. Then the split problem $\mathcal{M}$ is well-posed.

(2) Assume that the split problem $\mathcal{M}$ is well-posed. Moreover, assume that the following conditions hold:

(a) For all $\varepsilon>0$ and $u \in \Omega^{P}(\varepsilon)$ there exists $\sigma \in \Omega^{Q}(\varepsilon)$ such that $(u, \sigma) \in \Omega^{M}(\varepsilon)$.

(b) For all $\varepsilon>0$ and $\sigma \in \Omega^{Q}(\varepsilon)$ there exists $u \in \Omega^{P}(\varepsilon)$ such that $(u, \sigma) \in \Omega^{M}(\varepsilon)$.

Then, Problems $P$ and $Q$ are well-posed and their solutions, denoted by $u$ and $\sigma$, respectively, satisfy the equation $G(u, \sigma)=f$.

Proof (1) Let $x=(u, \sigma)$. Then, since $u \in S_{P}, \sigma \in S_{Q}$ and $G(u, \sigma)=f$, it follows from Definition 5 that $x \in S_{M}$ and, therefore

$$
S_{M} \neq \emptyset .
$$

On the other hand, since Problems $P$ and $Q$ are well-posed it follows from Theorem 3 that

$$
\operatorname{diam}\left(\Omega^{P}(\varepsilon)\right) \rightarrow 0 \quad \text { and } \quad \operatorname{diam}\left(\Omega^{P}(\varepsilon)\right) \rightarrow 0, \quad \text { as } \varepsilon \rightarrow 0 .
$$


Next, using the inclusion $\Omega^{M}(\varepsilon) \subset \Omega^{P}(\varepsilon) \times \Omega^{Q}(\varepsilon)$, the definition (2.1) of the norm in the space $\mathcal{X}$ and (2.4), it is easy to see that for each $\varepsilon>0$ we have

$$
\operatorname{diam}\left(\Omega^{M}(\varepsilon)\right) \leq \operatorname{diam}\left(\Omega^{P}(\varepsilon)\right)+\operatorname{diam}\left(\Omega^{Q}(\varepsilon)\right) .
$$

Therefore, (2.18) implies that

$$
\operatorname{diam}\left(\Omega^{M}(\varepsilon)\right) \rightarrow 0 \quad \text { as } \varepsilon \rightarrow 0
$$

Equations (2.17) and (2.19) allow us to use Theorem 10 in order to deduce that Problem $\mathcal{M}$ is well-posed.

(2) Assume now that the split problem $\mathcal{M}$ is well-posed and conditions (a), (b) above are satisfied. Then, it follows from Theorem 10 that $S_{M} \neq \emptyset$ and (2.19) holds. Therefore, there exists an element $x=(u, \sigma)$ such that

$$
u \in S_{P}, \sigma \in S_{Q}, \quad G(u, \sigma)=f .
$$

It follows that

$$
S_{P} \neq \varnothing
$$

and

$$
S_{Q} \neq \emptyset \text {. }
$$

Arguing by contradiction, we assume in what follows that $\operatorname{diam}\left(\Omega^{P}(\varepsilon)\right) \nrightarrow 0$ as $\varepsilon \rightarrow 0$. Then, there exist $\delta_{0} \geq 0$, a sequence $0<\varepsilon_{n} \rightarrow 0$ and two sequences $\left\{u_{n}\right\},\left\{v_{n}\right\} \subset X$ such that $u_{n}, v_{n} \in \Omega^{P}\left(\varepsilon_{n}\right)$ and

$$
\left\|u_{n}-v_{n}\right\|_{X} \geq \frac{\delta_{0}}{2} \quad \forall n \in \mathbb{N}
$$

We now use assumption (a) to deduce that there exist two sequences $\left\{\sigma_{n}\right\},\left\{\tau_{n}\right\} \subset Y$ such that $\left(u_{n}, \sigma_{n}\right) \in \Omega^{M}\left(\varepsilon_{n}\right)$ and $\left(v_{n}, \tau_{n}\right) \in \Omega^{M}\left(\varepsilon_{n}\right)$, for all $n \in \mathbb{N}$. Denote $x_{n}=\left(u_{n}, \sigma_{n}\right)$ and $y_{n}=$ $\left(v_{n}, \tau_{n}\right)$. Then, (2.1) and (2.23) imply that

$$
\left\|x_{n}-y_{n}\right\| \mathcal{X} \geq \frac{\delta_{0}}{2} \quad \forall n \in \mathbb{N}
$$

Now, since $\left\{x_{n}\right\} \in \Omega^{M}\left(\varepsilon_{n}\right)$ and $\left\{y_{n}\right\} \in \Omega^{M}\left(\varepsilon_{n}\right)$ it follows from (2.24) that

$$
\operatorname{diam}\left(\Omega^{M}(\varepsilon)\right) \geq \frac{\delta_{0}}{2},
$$

which is in contradiction with (2.19) due to the well-posedness of the split problem $\mathcal{M}$. We conclude from this that

$$
\operatorname{diam}\left(\Omega^{P}(\varepsilon)\right) \rightarrow 0 \quad \text { as } \varepsilon \rightarrow 0 .
$$


Using similar arguments and assumption (b) we deduce that

$$
\operatorname{diam}\left(\Omega^{Q}(\varepsilon)\right) \rightarrow 0 \quad \text { as } \varepsilon \rightarrow 0
$$

We now use (2.21), (2.25) and Theorem 10 to see that Problem $P$ is well-posed. Based on (2.22) and (2.26), a similar argument shows that Problem $Q$ is well-posed, too. We now recall equality $G(u, \sigma)=f$ in (2.20) to conclude the proof.

We end this section with the remark that some elementary examples of split problems $\mathcal{M}$ for which conditions (a) and (b) in Theorem 14 can be easily constructed.

Remark 15 Consider the Problems $P$ and $Q$ in Example 8 and recall that the solution of these problems are $u=1$ and $\sigma=2$, respectively. Moreover, using the inequalities (2.11) it is easy to see that these problems are well-posed. Assume now that $f=1$. Then, Theorem 14(1) implies that the corresponding split problem $\mathcal{M}$ is well-posed. This result recovers one of the implication of the Claim 1 in Example 8.

\section{A split variational-hemivariational inequality}

In this section we use Theorem 13 in the study of the well-posedness for a split variationalhemivariational inequality. The problem under consideration is of the form (2.6) and, to introduce it, we start by introducing the problems $P$ and $Q$ together with the families of approximating sets $\left\{\Omega^{P}(\varepsilon)\right\}_{\varepsilon>0}$ and $\left\{\Omega^{Q}(\varepsilon)\right\}_{\varepsilon>0}$, respectively.

Denote by $X^{*}$ and $\langle\cdot, \cdot\rangle$ the dual of the space $X$ and the duality pairing between $X^{*}$ and $X$. Let $U \subset X, A: X \rightarrow X^{*}, \varphi: X \times X \rightarrow \mathbb{R}, j: X \rightarrow \mathbb{R}$ and $p \in X^{*}$. We assume that $j$ is a locally Lipshitz function and denote by $j^{0}(u ; v)$ the Clarke generalized directional derivative of $j$ at the point $u \in X$ in the direction $v \in X$. Then, Problem $P$ concerns the following variational-hemivariational inequality.

Problem $P$ Find $u$ such that

$$
\begin{gathered}
u \in U, \quad\langle A u, v-u\rangle+\varphi(u, v)-\varphi(u, u)+j^{0}(u ; v-u) \\
\geq\langle p, v-u\rangle \quad \forall v \in U .
\end{gathered}
$$

A solution for Problem $P$ is an element $u$ which solves (3.1). As usual, we denote in what follows by $S_{P}$ the set of solutions to Problem $P$. Moreover, for each $\varepsilon>0$ we consider the set $\Omega^{P}(\varepsilon)$ defined as follows:

$$
\begin{aligned}
\Omega^{P}(\varepsilon)= & \{u \in U:\langle A u-p, v-u\rangle+\varphi(u, v)-\varphi(u, u) \\
& \left.+j^{0}(u ; v-u) \geq-\varepsilon\|u-v\|_{X} \forall v \in U\right\} .
\end{aligned}
$$

Note that condition (2.3) is satisfied.

We now move to Problem $Q$ which is again a variational-hemivariational inequality, defined as follows. 
Problem $Q$ Find $\sigma$ such that

$$
\begin{gathered}
\sigma \in \Sigma, \quad\langle\langle B \sigma, \tau-\sigma\rangle\rangle+\phi(\sigma, \tau)-\phi(\sigma, \sigma)+k^{0}(\sigma ; \tau-\sigma) \\
\geq\langle\langle q, \tau-\sigma\rangle\rangle \quad \forall \tau \in \Sigma .
\end{gathered}
$$

Here $Y^{*}$ is the dual of the space $Y$ and $\langle\langle\cdot, \cdot\rangle\rangle$ represents the duality pairing between $Y^{*}$ and $Y$. Moreover, $\Sigma \subset Y, B: Y \rightarrow Y^{*}, \phi: Y \times Y \rightarrow \mathbb{R}, q \in Y^{*}, k: Y \rightarrow \mathbb{R}$ is a locally Lipshitz function and $k^{0}(\sigma ; \tau)$ stands for the Clarke generalized directional derivative of $k$ at the point $\sigma \in Y$ in the direction $\tau \in Y$. We denote by $S_{Q}$ the set of solutions to $Q$ and, for each $\varepsilon>0$, we consider the set $\Omega^{Q}(\varepsilon)$ defined as follows:

$$
\begin{aligned}
\Omega^{Q}(\varepsilon)= & \{\sigma \in \Sigma:\langle\langle B \sigma-q, \tau-\sigma\rangle)+\phi(\sigma, \tau)-\phi(\sigma, \sigma) \\
& \left.+k^{0}(\sigma ; \tau-\sigma) \geq-\varepsilon\|\tau-\sigma\|_{Y} \forall \tau \in \Sigma\right\} .
\end{aligned}
$$

Note that condition (2.5) is, obviously, satisfied.

Then, given $G: X \times Y \rightarrow Z$ and $f \in Z$, the split problem under consideration is the following.

Problem $\mathcal{M}$ Find $x=(u, \sigma)$ such that $u$ is a solution of (3.1), $\sigma$ is a solution of (3.3) and $G(u, \sigma)=f$.

Note that, since both Problems $P$ and $Q$ are variational-hemivariational inequalities, we refer to the split problem $\mathcal{M}$ as a split variational-hemivariational inequality.

We now study the well-posedness of this split variational-hemivariational inequality and, to this end, we consider the following assumptions concerning Problem $P$.

$U$ is a nonempty closed convex subset of $X$.

$A: X \rightarrow X^{*}$ is a monotone hemicontinuous operator.

$\varphi(u, \cdot): X \rightarrow \mathbb{R}$ is a convex function, for all $u \in X$.

$\left\{\begin{array}{l}\varphi: X \times X \rightarrow \mathbb{R} \text { and, for all sequences }\left\{u_{n}\right\},\left\{v_{n}\right\} \\ \quad \text { such that } u_{n} \rightarrow u \text { in } X, v_{n} \rightarrow v \text { in } X, \text { we have } \\ \lim \sup \left(\varphi\left(u_{n}, v_{n}\right)-\varphi\left(u_{n}, u_{n}\right)\right) \leq \varphi(u, v)-\varphi(u, u) .\end{array}\right.$

$j: X \rightarrow \mathbb{R}$ is a locally Lipschitz function.

$p \in X^{*}$.

Recall that an operator $A: X \rightarrow X^{*}$ is said to be monotone, if for all $u, v \in X$, we have $\langle A u-A v, u-v\rangle \geq 0$. The operator $A$ is said to be hemicontinuous if for all $u, v, w \in X$, the function $\lambda \mapsto\langle A(u+\lambda v), w\rangle$ is continuous on $[0,1]$. A function $j: X \rightarrow \mathbb{R}$ is said to be locally Lipschitz, if for every $u \in X$, there exist $N_{u}$ a neighborhood of $u$ and a constant $L_{u}>0$ such that

$$
|j(x)-j(y)| \leq L_{x}\|x-y\|_{X}
$$


for all $x, y \in N_{u}$. Moreover, the Clarke generalized directional derivative of $j$ at the point $u \in X$ in the direction $v \in X$ is defined by

$$
j^{0}(u ; v)=\limsup _{x \rightarrow u, \lambda \downarrow 0} \frac{j(x+\lambda v)-j(x)}{\lambda} .
$$

Details on the definitions above can be found in $[7,24,30]$, for instance.

We proceed with the following assumptions which concern Problem $Q$ :

$\Sigma$ is a nonempty closed convex subset of $Y$.

$B: Y \rightarrow Y^{*}$ is a monotone hemicontinuous operator.

$\phi(\sigma, \cdot): Y \rightarrow \mathbb{R}$ is a convex function, for all $\sigma \in Y$.

$\phi \phi: Y \times Y \rightarrow \mathbb{R}$ and, for all sequences $\left\{\sigma_{n}\right\},\left\{\tau_{n}\right\}$

such that $\sigma_{n} \rightarrow \sigma$ in $Y, \tau_{n} \rightarrow \tau$ in $Y$, we have

$\lim \sup \left(\phi\left(\sigma_{n}, \tau_{n}\right)-\phi\left(\sigma_{n}, \sigma_{n}\right)\right) \leq \phi(\sigma, \tau)-\varphi(\sigma, \sigma)$.

$k: Y \rightarrow \mathbb{R}$ is a locally Lipschitz function.

$q \in Y^{*}$.

Finally, we consider the following assumptions on $G$ and $f$ :

$G: X \times Y \rightarrow Z$ is a continuous operator.

$f \in Z$.

Our result in the study of Problem $\mathcal{M}$ is the following.

Theorem 16 Assume that $X$ and $Y$ are Banach spaces and (3.5)-(3.18) hold. Then, the split variational-hemivariational inequality $\mathcal{M}$ is well-posed if and only iffor each $\varepsilon>0$ the set $\Omega(\varepsilon)$ is nonempty and $\operatorname{diam}\left(\Omega^{M}(\varepsilon)\right) \rightarrow 0$ as $\varepsilon \rightarrow 0$.

Proof We claim that the family of approximating sets $\left\{\Omega^{M}(\varepsilon)\right\}_{\varepsilon>0}$ defined by (2.8), (3.2) and (3.4) is regular in the sense of Definition 12. First, it is easy to see that condition (a) holds. Consider now an approximating sequence $\left\{u_{n}\right\}$ of Problem $P$ which converges to an element $u \in X$. This implies that $u_{n} \in \Omega^{P}\left(\varepsilon_{n}\right)$ with $0<\varepsilon_{n} \rightarrow 0$. Therefore, the regularity $u_{n} \in U$ in (3.1) and assumption (3.5) yield

$$
u \in U \text {. }
$$

Moreover, for any $n \in \mathbb{N}$, we have

$$
\begin{aligned}
& \left\langle A u_{n}, v-u_{n}\right\rangle+\varphi\left(u_{n}, v\right)-\varphi\left(u_{n}, u_{n}\right)+j^{0}\left(u_{n} ; v-u_{n}\right) \\
& \geq\left\langle f, v-u_{n}\right\rangle-\varepsilon_{n}\left\|u_{n}-v\right\|_{X} \quad \forall v \in U
\end{aligned}
$$


and, using the monotonicity of the operator $A$, we deduce that

$$
\begin{aligned}
& \left\langle A v, v-u_{n}\right\rangle+\varphi\left(u_{n}, v\right)-\varphi\left(u_{n}, u_{n}\right)+j^{0}\left(u_{n} ; v-u_{n}\right) \\
& \geq\left\langle f, v-u_{n}\right\rangle-\varepsilon_{n}\left\|u_{n}-v\right\|_{X} \quad \forall v \in U .
\end{aligned}
$$

Note that the upper semicontinuity of the Clarke generalized directional derivative (see [30, Proposition 71], for instance) combined with the convergence $u_{n} \rightarrow u$ in $X$ yields

$$
\lim \sup j^{0}\left(u_{n} ; v-u_{n}\right) \leq j^{0}(u ; v-u) .
$$

We now pass to the upper limit as $n \rightarrow \infty$ in (3.20) and use inequality (3.21), the convergences $\varepsilon_{n} \rightarrow 0, u_{n} \rightarrow u$ in $X$, together with assumption (3.8), to obtain

$$
\langle A v, v-u\rangle+\varphi(u, v)-\varphi(u, u)+j^{0}(u ; v-u) \geq\langle f, v-u\rangle \quad \forall v \in U
$$

Let $w \in U$ and $t \in(0,1]$. We test by the previous inequality with $v=u+t(w-u) \in U$, use assumption (3.7), the positivity homogeneity of the Clarke generalized directional derivative $\left(j^{0}(u ; \lambda v)=\lambda j^{0}(u ; v)\right.$ for all $\left.v \in V, \lambda \geq 0\right)$, and divide the resulting inequality with $t$ to find that

$$
\begin{aligned}
& \langle A(u+t(w-u)), w-u\rangle+\varphi(u, w)-\varphi(u, u)+j^{0}(u ; w-u) \\
& \quad \geq\langle f, w-u\rangle \quad \forall w \in U .
\end{aligned}
$$

Finally, we pass to the limit as $t \downarrow 0$ and use the hemicontinuity of the operator $A$ to see that

$$
\begin{aligned}
& \langle A u, w-u\rangle+\varphi(u, w)-\varphi(u, u)+j^{0}(u ; w-u) \\
& \geq\langle f, w-u\rangle \quad \forall w \in U .
\end{aligned}
$$

We now combine (3.19) and (3.22) to see that $u$ is a solution to the variationalhemivariational inequality (3.1). This shows that condition (b) in Definition 12 is satisfied.

Based on assumptions (3.11)-(3.16), similar arguments show that condition (c) in Definition 12 is satisfied, too. Therefore, the family $\left\{\Omega^{M}(\varepsilon)\right\}_{\varepsilon>0}$ is regular, as claimed. We are now in a position to apply Theorem 13 to conclude the proof.

Note that Theorem 16 provides only an equivalence result. Its statement does not guarantee that the split problem $\mathcal{M}$ is well-posed. Sufficient conditions which guarantee its well-posedness can be considered, based on the existence and uniqueness results for variational-hemivariational inequalities obtained in [22,30]. They involve the strong monotonicity of the operator $A$, the relaxed monotonicity condition for the functions $j$ as well as a smallness condition related to $A, \varphi$ and $j$.

We end this section with an elementary example in which Theorem 16 can be used. 
Example 17 Let $X=Y=Z=\mathbb{R}$, let $a, b, c, d, p, q \in \mathbb{R}$ be such that $a<b, c<d, p \in(a, b)$, $q \in(c, d)$ and consider the split problem $\mathcal{M}=\mathcal{M}(P, Q, G, f)$ defined as follows.

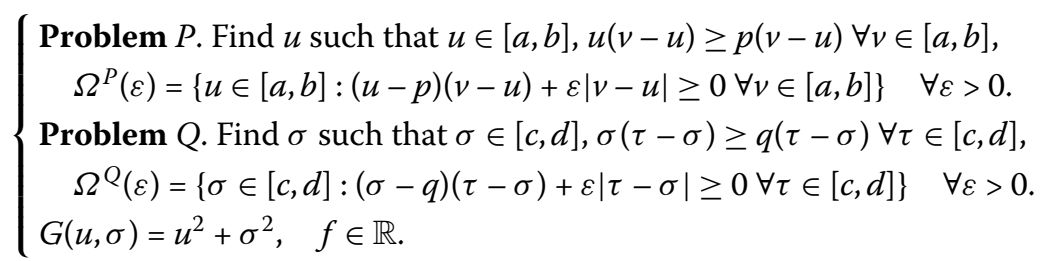

Note that Problem $\mathcal{M}$ is a particular case of split variational-hemivariational inequality studied above in this section, obtained when functions $\varphi, \phi, j$ and $k$ vanish. Moreover, using the properties of the projections it is easy to see that in this case $S_{P}=\{p\}$ and $S_{Q}=\{q\}$. In addition, we have the following result.

Claim 2 The split problem $\mathcal{M}$ is well-posed if and only if $p^{2}+q^{2}=f$.

Proof Assume that $\mathcal{M}$ is well-posed. Then using Definitions 7 and 5 it follows that $G(p, q)=f$ and, therefore, $p^{2}+q^{2}=f$.

Conversely, assume that $p^{2}+q^{2}=f$. Then it follows that Problem $\mathcal{M}$ has a unique solution $x=(p, q)$. Let $\varepsilon>0$ and let $u \in \mathbb{R}$ be such that

$$
(u-p)(v-u)+\varepsilon|v-u| \geq 0 \quad \forall v \in[a, b] .
$$

Then, taking $v=p$ we deduce that

$$
|u-p|<\varepsilon \text {. }
$$

On the other hand, if (3.25) holds we find that $|(u-p)(v-u)| \leq \varepsilon|v-u|$ for all $v \in \mathbb{R}$, which implies (3.24). We conclude from above that inequalities (3.24) and (3.25) are equivalent and, therefore, since $p \in(a, b)$, we deduce that for $\varepsilon$ small enough we have $\Omega^{P}(\varepsilon)=$ $[p-\varepsilon, p+\varepsilon]$. Similar arguments show that for $\varepsilon$ small enough we have $\Omega^{Q}(\varepsilon)=[q-\varepsilon, q+\varepsilon]$. We now use definition $(2.8)$ to see that in this case $\Omega^{M}(\varepsilon) \subset[p-\varepsilon, p+\varepsilon] \times[q-\varepsilon, q+\varepsilon]$ and, therefore, $\operatorname{diam}\left(\Omega^{M}(\varepsilon)\right) \rightarrow 0$ as $\varepsilon \rightarrow 0$. Theorem 16 guarantees now that Problem $\mathcal{M}$ is well-posed, which concludes the proof of the claim.

\section{A well-posed split variational inequality}

In this section we present an example of well-posed split variational inequality in Hilbert spaces. To this end we shall use the abstract framework in Sect. 2 in the particular case when $X=Y=Z$ and, therefore, $\mathcal{X}=X \times X$. We denote by $(\cdot, \cdot)_{X}$ and $0_{X}$ the inner product and the zero element of the space $X$, respectively, and we consider a strongly monotone Lipschitz continuous operator $A$ on $X$, i.e.,

$$
\left\{\begin{array}{l}
A: X \rightarrow X \text { is such that } \\
\text { (a) there exists } m_{A}>0 \text { such that } \\
\quad\left(A v_{1}-A v_{2}, v_{1}-v_{2}\right)_{X} \geq m_{A}\left\|v_{1}-v_{2}\right\|_{X}^{2} \quad \forall v_{1}, v_{2} \in X ; \\
\text { (b) there exists } L_{A}>0 \text { such that } \\
\left\|A v_{1}-A v_{2}\right\|_{X} \leq L_{A}\left\|v_{1}-v_{2}\right\|_{X} \quad \forall v_{1}, v_{2} \in X .
\end{array}\right.
$$


Recall that, since $A$ is strongly monotone Lipschitz continuous on $X$, it follows that $A$ is invertible and its inverse $A^{-1}: X \rightarrow X$ is strongly monotone and Lipschitz continuous, too. A proof of this result can be found in [29, p.23]. Moreover, consider a function $j$ such that

$$
\left\{\begin{array}{l}
j: X \rightarrow \mathbb{R}_{+} \text {is convex, lower semicontinuous and } \\
\text { positively homogenous, i.e., } j(\lambda v)=\lambda j(v) \forall v \in X, \lambda \geq 0 .
\end{array}\right.
$$

Note that any continuous seminorm on the space $X$ satisfies assumption (4.2). Finally, assume that $p \in X$ and consider the set $\Sigma$, which depends on $j$ and $p$, defined by

$$
\Sigma=\left\{\tau \in X:(\tau, v)_{X}+j(v) \geq(p, v)_{X} \forall v \in X\right\}
$$

With these data we consider the following problem.

Problem $\mathcal{M}$ Find $x=(u, \sigma)$ such that

$$
\begin{aligned}
& u \in X, \quad(A u, v-u)_{X}+j(v)-j(u) \geq(p, v-u)_{X} \quad \forall v \in X, \\
& \sigma \in \Sigma, \quad\left(A^{-1} \sigma, \tau-\sigma\right)_{X} \geq 0 \quad \forall \tau \in \Sigma, \\
& \sigma=A u .
\end{aligned}
$$

It is easy to see that Problem $\mathcal{M}$ is a split variational inequality of the form $\mathcal{M}=$ $\mathcal{M}(P, Q, G, f)$ in which Problem $P$ is given by the variational inequality (4.4), Problem $Q$ is given by the variational inequality (4.5), $G(u, \sigma)=A u-\sigma$ and $f=0_{X}$.

The sets of solutions to inequalities (4.4) and (4.5) will be denoted by $S_{P}$ and $S_{Q}$, as usual. Moreover, the approximating sets of these problems are given by

$$
\begin{aligned}
& \Omega^{P}(\varepsilon)=\left\{u \in X:(A u-p, v-u)_{X}+j(v)-j(u) \geq-\varepsilon\|u-v\|_{X} \forall v \in X\right\} \\
& \Omega^{Q}(\varepsilon)=\left\{\sigma \in \Sigma:\left(A^{-1} \sigma, \tau-\sigma\right)_{X} \geq-\varepsilon\|\sigma-\tau\|_{X} \forall \tau \in \Sigma\right\}
\end{aligned}
$$

for each $\varepsilon>0$. Therefore, using (2.8) we have

$$
\Omega^{M}(\varepsilon)=\left\{x=(u, \sigma): u \in \Omega^{P}(\varepsilon), \sigma \in \Omega^{Q}(\varepsilon),\|A u-\sigma\|_{X} \leq \varepsilon\right\} .
$$

In addition, using notation (2.7) we see that condition (2.9) is, obviously, satisfied.

Our result in the study of Problem $P$ is the following.

Theorem 18 Assume (4.1) and (4.2). Then, for each $p \in X$ the split variational inequality $\mathcal{M}$ is well-posed.Moreover, the operator $p \mapsto x(p)$ which maps every $p \in X$ into the solution of Problem $\mathcal{M}$ is Lipschitz continuous.

Proof We shall use Theorem 14 and, to this end, we start by proving the well-posedness of the variational inequalities (4.4) and (4.5) for an arbitrary $p \in X$, assumed in what follows to be given.

First, we note that a standard result on variational inequalities ([29, Theorem 2.8], for instance) guarantees that, under assumptions (4.1) and (4.2), problem (4.4) has a unique solution. Assume now that $\left\{u_{n}\right\} \subset X$ is an approximating sequence for (4.4), i.e. $u_{n} \in \Omega^{P}\left(\varepsilon_{n}\right)$ 
with $0<\varepsilon_{n} \rightarrow 0$. Let $n \in \mathbb{N}$. We use (4.4) with $v=u_{n}$ and (4.7) with $v=u$ to see that

$$
\begin{aligned}
& \left(A u, u_{n}-u\right)_{X}+j\left(u_{n}\right)-j(u) \geq\left(p, u_{n}-u\right)_{X}, \\
& \left(A u_{n}, u-u_{n}\right)_{X}+j(u)-j\left(u_{n}\right) \geq\left(p, u-u_{n}\right)_{X}-\varepsilon_{n}\left\|u_{n}-u\right\|_{X} .
\end{aligned}
$$

We now add these inequalities to find that

$$
\left(A u_{n}-A u, u_{n}-u\right)_{X} \leq \varepsilon_{n}\left\|u_{n}-u\right\|_{X}
$$

then we use assumption (4.1)(a) to obtain

$$
\left\|u_{n}-u\right\|_{X} \leq \frac{\varepsilon_{n}}{m_{A}} .
$$

Finally, since $\varepsilon_{n} \rightarrow 0$, we deduce that $u_{n} \rightarrow u$ in $X$. Therefore, using Definition 2 we deduce that the variational inequality (4.4) is well-posed.

Next, we claim that the set $\Sigma$ defined by (4.3) is nonempty. Indeed, assumption (4.2) guarantees that $j$ is a subdifferentiable function on $X$ and, therefore there exists an element $\theta$ such that $\theta \in \partial j\left(0_{X}\right)$. Note that here and everywhere in this section we use the notion of subdifferentiability in the sense of the convex analysis; see for instance $[9,18]$. Therefore, for $z \in X$, notation $\partial j(z)$ represents the subgradient of $j$ in $z$ defined by

$$
\partial j(z)=\left\{\xi \in X: j(v)-j(z) \geq(\xi, v-z)_{X} \forall v \in X\right\} .
$$

Since $j\left(0_{X}\right)=0$, inclusion $\theta \in \partial j\left(0_{X}\right)$ yields $j(v) \geq(\theta, v)_{X}$ for any $v \in X$, which implies that the element $\tau=p-\theta$ belongs to $\Sigma$ and proves the claim. On the other hand, it is easy to see that $\Sigma$ is a convex closed subset of $X$. Therefore, recalling that $A^{-1}: X \rightarrow X$ is a strongly monotone Lipschitz continuous operator, we deduce that the arguments used in the study of the variational inequality (4.4) can be used in the study of the variational inequality (4.5), too. We conclude from this that the variational inequality (4.5) is well-posed. In particular, this inequality has a unique solution that we denote in what follows by $\sigma$.

Let $u \in X$ be the solution of inequality (4.4) and, moreover, let $\widetilde{\sigma}=A u$, which implies that $A^{-1} \widetilde{\sigma}=u$. Then,

$$
(\widetilde{\sigma}, v-u)_{X}+j(v)-j(u) \geq(p, v-u)_{X} \quad \forall v \in X .
$$

We now take $v=2 u$ and $v=0_{X}$ in (4.10) and use assumption (4.2) to deduce that

$$
(\widetilde{\sigma}, u)_{X}+j(u)=(p, u)_{X}
$$

Next, we combine Eqs. (4.10) and (4.11) to see that

$$
(\widetilde{\sigma}, v)_{X}+j(v) \geq(p, v)_{X} \quad \forall v \in X,
$$

which shows that

$$
\tilde{\sigma} \in \Sigma \text {. }
$$


Let $\tau \in X$. Then, using equality $A^{-1} \widetilde{\sigma}=u$ and (4.11) we find that

$$
\left(A^{-1} \widetilde{\sigma}, \tau-\widetilde{\sigma}\right)_{X}=(\tau-\widetilde{\sigma}, u)_{X}=(\tau, u)_{X}+j(u)-(p, u)_{X}
$$

and, using definition (4.3) we deduce that

$$
\left(A^{-1} \widetilde{\sigma}, \tau-\widetilde{\sigma}\right)_{X} \geq 0 \quad \forall \tau \in \Sigma .
$$

We now combine Eqs. (4.12) and (4.13) to see that $\widetilde{\sigma}$ is a solution to the variational inequality (4.5). On the other hand, recall that this inequality has a unique solution, denoted by $\sigma$. It follows from this that $\widetilde{\sigma}=\sigma$ and, therefore, $\sigma=A u$.

To conclude, we proved that inequalities (4.4) and (4.5) are well-posed and their solutions are such that $\sigma=A u$. We are now in a position to use Theorem 141) to conclude that the split variational inequality $\mathcal{M}$ is well-posed, which concludes the first part of the proof.

For the second part, assume that $p, p^{\prime} \in X$ and, for simplicity, denote $x(p)=(u, \sigma), x\left(p^{\prime}\right)=$ $\left(u^{\prime}, \sigma^{\prime}\right)$. We use (4.4) to see that

$$
\begin{aligned}
& (A u, v-u)_{X}+j(v)-j(u) \geq(p, v-u)_{X} \\
& \left(A u^{\prime}, v-u^{\prime}\right)_{X}+j(v)-j\left(u^{\prime}\right) \geq\left(p^{\prime}, v-u^{\prime}\right)_{X}
\end{aligned}
$$

for all $v \in X$. We now take $v=u^{\prime}$ in the first inequality and $v=u$ in the second one, then we add the resulting inequalities to obtain

$$
\left(A u^{\prime}-A u, u^{\prime}-u\right)_{X} \leq\left(p^{\prime}-p, u^{\prime}-u\right)_{X} \leq\left\|p^{\prime}-p\right\|_{X}\left\|u_{n}-u\right\|_{X} .
$$

This inequality combined with assumption (4.1)(a) yields

$$
\left\|u^{\prime}-u\right\|_{X} \leq \frac{1}{m_{A}}\left\|p^{\prime}-p\right\|_{X} .
$$

Moreover, using (4.6) we obtain $\sigma=A u, \sigma^{\prime}=A u^{\prime}$ and, therefore, (4.14) combined with assumption (4.1)(b) implies that

$$
\left\|\sigma^{\prime}-\sigma\right\|_{X} \leq \frac{L_{A}}{m_{A}}\left\|p^{\prime}-p\right\|_{X}
$$

The Lipschitz continuity of the operator $p \mapsto x(p): X \rightarrow \mathcal{X}$ is now a direct consequence of (4.14), (4.15) and (2.1).

We end this section with the remark that split variational inequalities of the form (4.4)(4.6) arise in the study of static frictional contact problems with elastic materials. There, $u$ represents the displacement field and $\sigma$ is related to the stress field. The operator $A$ describes the properties of the material, the function $j$ is related to the frictional contact conditions and $p$ is determined by the external forces and tractions acting on the body. Inequality (4.4) represents the variational formulation of the contact problem in terms of displacements, the so-called primal variational formulation. Inequality (4.5) represents the 
variational formulation of the contact problem in terms of stress, the so-called dual variational formulation. Finally, Eq. (4.5) is related to the constitutive law of the material. More details, comments and physical interpretation on such kind of problems can be found in $[4,8,29]$. Here we restrict ourselves to the following example.

Example 19 Assume that $D$ is a regular domain in $\mathbb{R}^{2}$ with smooth boundary $\partial D=\Gamma$, composed of three measurable sets $\bar{\Gamma}_{1}, \bar{\Gamma}_{2}$ and $\bar{\Gamma}_{3}$, with the mutually disjoint relatively open sets $\Gamma_{1}, \Gamma_{2}$ and $\Gamma_{3}$, such that the one-dimensional measure of $\Gamma_{1}$ is strictly positive. Consider the following boundary value problem.

Problem $P_{\text {ant }}$ Find a displacement field $u: D \rightarrow \mathbb{R}$ and a stress field $\widetilde{\sigma}: D \rightarrow \mathbb{R}^{2}$ such that

$$
\begin{aligned}
& \tilde{\sigma}=\mu \nabla u \quad \text { in } D, \\
& \operatorname{div} \widetilde{\sigma}+f_{0}=0 \quad \text { in } D, \\
& u=0 \quad \text { on } \Gamma_{1}, \\
& \tilde{\sigma} \cdot v=f_{2} \quad \text { on } \Gamma_{2}, \\
& |\widetilde{\sigma} \cdot v| \leq g, \quad \tilde{\sigma} \cdot v=-g \frac{u}{|u|} \quad \text { if } u \neq 0 \text { on } \Gamma_{3} .
\end{aligned}
$$

Problem $P_{\text {ant }}$ is a so-called antiplane contact problem see, for instance, [28, Sect. 9.1]. It describes the equilibrium of an elastic cylinder of transversal section $D$ under the action of axial body forces of density $f_{0}$ and surface traction of density $f_{2}$. Equation (4.16) is the constitutive law in which $\mu$ denotes the Lamé coefficient, (4.17) represents the equation of equilibrium, (4.18) is the displacement boundary condition and (4.19) represents the traction boundary condition. Here and below $v$ is the unit outward vector to $\Gamma$ and "." is the inner product on $\mathbb{R}^{2}$. Finally, (4.20) is a static version of Coulomb's law in which $g$ is a positive function, the friction bound. Note that in (4.16)-(4.20) and below we skip the dependence of various functions with respect to the spatial variable $\boldsymbol{x} \in D \cup \Gamma$.

For the variational analysis of Problem $P_{\text {ant }}$ we use the space $X$ given by

$$
X=\left\{v \in H^{1}(D): v=0 \text { on } \Gamma_{1}\right\}
$$

and we denote by $(\cdot, \cdot)_{X}$ and $\|\cdot\|_{X}$ the inner product on $X$ induced by the inner product of $H^{1}(D)$ and the associated norm, respectively. Moreover, we use $0_{X}$ for the zero element of $X$ and we assume that the data satisfy the following conditions:

$$
\begin{aligned}
& \mu \in L^{\infty}(D) \text { and there exists } \mu^{*}>0 \quad \text { such that } \\
& \mu(\boldsymbol{x}) \geq \mu^{*} \quad \text { a.e. } \boldsymbol{x} \in D . \\
& f_{0} \in L^{2}(D), \quad f_{2} \in L^{2}\left(\Gamma_{2}\right) . \\
& g \in L^{\infty}\left(\Gamma_{3}\right) \quad \text { and } \quad g(\boldsymbol{x}) \geq 0 \quad \text { a.e. } \boldsymbol{x} \in \Gamma_{3} .
\end{aligned}
$$


Then, using standard arguments, it is easy to see that

$$
\begin{gathered}
\int_{D} \tilde{\sigma} \cdot(\nabla v-\nabla u) d x+\int_{\Gamma_{3}} g|v| d a-\int_{\Gamma_{3}} g|u| d a \\
\geq \int_{D} f_{0}(v-u) d x+\int_{\Gamma_{2}} f_{2}(v-u) d a \quad \forall v \in X .
\end{gathered}
$$

Define now the operator $A: X \rightarrow X$, the function $j: X \rightarrow \mathbb{R}$, the element $p \in X$ and the set $\Sigma \subset X$ by equalities

$$
\begin{aligned}
& (A u, v)_{X}=\int_{D} \mu \nabla u \cdot \nabla v d x \quad \forall u, v \in X, \\
& j(v)=\int_{\Gamma_{3}} g|v| d a \quad \forall v \in X, \\
& (p, v)_{X}=\int_{D} f_{0} v d x+\int_{\Gamma_{2}} f_{2} v d a \quad \forall v \in X, \\
& \Sigma=\left\{\tau \in X:(\tau, v)_{X}+j(v) \geq(p, v)_{X} \forall v \in X\right\} .
\end{aligned}
$$

Then, using (4.16), (4.24)-(4.27), we derive the following variational formulation of Problem $P_{\text {ant }}$.

Problem $P$ Find $u$ such that

$$
u \in X, \quad(A u, v-u)_{X}+j(v)-j(u) \geq(p, v-u)_{X} \quad \forall v \in X
$$

Next, we use the Riesz representation theorem to introduce the element $\sigma \in X$ related to the stress field $\widetilde{\sigma}$ by equality

$$
(\sigma, v)_{X}=\int_{D} \tilde{\sigma} \cdot \nabla v d x \quad \forall v \in X
$$

Note that (4.30), (4.16) and (4.25) imply that $\sigma=A u$ and, therefore, testing in (4.29) with $v=2 u$ and $v=0_{X}$ we deduce that

$$
(\sigma, v)_{X}+j(v) \geq(p, v)_{X} \quad \forall v \in X, \quad(\sigma, u)_{X}+j(u)=(p, u)_{X}
$$

We now combine (4.31) and (4.28) to see that

$$
\sigma \in \Sigma, \quad(u, \tau-\sigma)_{X} \geq 0 \quad \forall \tau \in \Sigma
$$

Note that the operator $A$ is inversible and, therefore, equality $\sigma=A u$ yields $u=A^{-1} \sigma$. We now substitute this equality in (4.32) to deduce the following variational formulation of Problem $P_{\text {ant }}$.

Problem $Q$ Find $\sigma$ such that

$$
\sigma \in \Sigma, \quad\left(A^{-1} \sigma, \tau-\sigma\right)_{X} \geq 0 \quad \forall \tau \in \Sigma
$$


Problems $P$ and $Q$ represent the primal and dual variational formulations of the antiplane contact problem $P_{\text {ant }}$, respectively. Recall that the solutions of these problems are related by the equality $\sigma=A u$. Therefore, gathering these ingredients, it is natural to consider the following split variational inequality.

Problem $\mathcal{M}$ Find $x=(u, \sigma)$ such that $u$ is a solution of (4.29), $\sigma$ is a solution of (4.33) and $\sigma=A u$.

Note that, under the assumption (4.22)-(4.24) we are in a position to a apply Theorem 18. Hence, we deduce that Problem $\mathcal{M}$ is well-posed and its solution depends Lipschitz-continuously on the density of body forces and surface tractions.

\section{A split history-dependent variational inequality}

In this section we present an example of well-posed split variational inequality with history-dependent operators in reflexive Banach spaces. To this end we start by recalling the definition of history-dependent operators.

Let $T>0$, let $\left(U,\|\cdot\|_{U}\right),\left(V,\|\cdot\|_{V}\right)$ be real normed spaces and denote by $C([0, T] ; U)$, $C([0, T] ; V)$ the spaces of continuous functions on $[0, T]$ with values in $U$ and $V$, respectively. An operator $\Lambda: C([0, T] ; U) \rightarrow C([0, T] ; V)$ is said to be history-dependent if there exists $L_{\Lambda}>0$ such that

$$
\left\{\begin{array}{c}
\left\|\Lambda u_{1}(t)-\Lambda u_{2}(t)\right\|_{V} \leq L_{\Lambda} \int_{0}^{t}\left\|u_{1}(s)-u_{2}(s)\right\|_{U} d s \\
\forall u_{1}, u_{2} \in C([0, T] ; U), t \in[0, T]
\end{array}\right.
$$

Moreover, we recall that the norm on the space $C([0, T] ; U)$ is given by

$$
\|u\|_{C([0, T] ; U)}=\max _{t \in[0, T]}\|u(t)\|_{U}
$$

Below in this section we shall use history-dependent operators in the case when $U=X$, $V=X^{*}$, and $U=X^{*}, V=X$, where $X^{*}$ represents the dual of $X$. Everywhere below we denote by $\|\cdot\|_{X^{*}}$ and $\langle\cdot, \cdot\rangle$ the norm on the space $X^{*}$ and the duality pairing between $X^{*}$ and $X$.

The split problem we consider is governed by a set $K \subset X$, two operators $A: X \rightarrow X^{*}$ and $B:[0, T] \times X \times X^{*} \rightarrow X^{*}$, and a function $p:[0, T] \rightarrow X^{*}$, which are assumed to satisfy the following conditions:

$K$ is a nonempty, convex, and closed set of $X$.

There exists an element $\widetilde{g}$ such that

(a) $\tilde{g} \in K$;

(b) $2 v-\tilde{g} \in K \quad \forall v \in K$.

$\left\{\begin{array}{l}A: X \rightarrow X^{*} \text { is a linear continuous and positively defined operator, } \\ \text { i.e., there exists } m_{A}>0 \text { such that }\langle A u, u\rangle \geq m_{A}\|u\|_{X}^{2} \forall u \in X .\end{array}\right.$ 


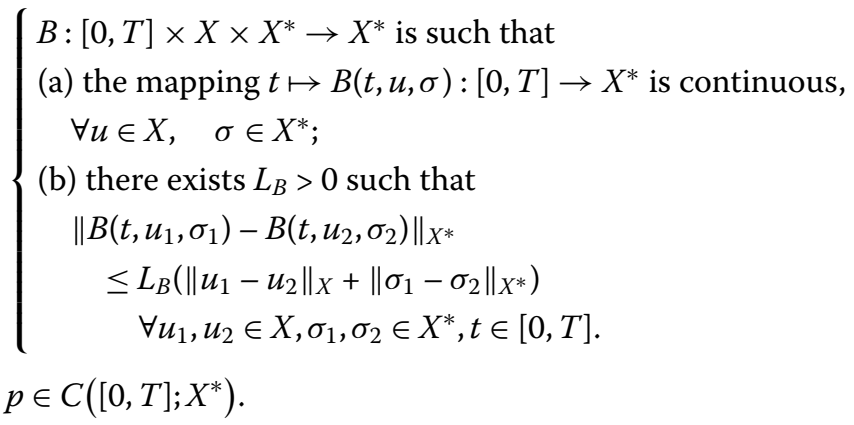

Note that the assumption (5.5) implies that the operator $A$ is invertible and its inverse $A^{-1}: X^{*} \rightarrow X$ is linear, positively defined, and satisfies the inequality

$$
\left\|A^{-1} \sigma\right\|_{X} \leq \frac{1}{m_{A}}\|\sigma\|_{X^{*}} \quad \forall \sigma \in X^{*} .
$$

Next, consider the implicit integral equation

$$
\sigma(t)=A u(t)+\int_{0}^{t} B(s, u(s), \sigma(s)) d s \quad \forall t \in[0, T]
$$

for which we recall the following result.

Theorem 20 Assume that $X$ is a Banach space and (5.5) and (5.6) hold. Then:

(1) There exists a history-dependent operator $\mathcal{S}: C([0, T] ; X) \rightarrow C\left([0, T] ; X^{*}\right)$ such that, for all functions $u \in C([0, T] ; X)$ and $\sigma \in C\left([0, T] ; X^{*}\right)$, equality (5.9) holds if and only if

$$
\sigma(t)=A u(t)+\mathcal{S} u(t) \quad \forall t \in[0, T]
$$

(2) There exists a history-dependent operator $\mathcal{R}: C\left([0, T] ; X^{*}\right) \rightarrow C([0, T] ; X)$ such that, for all functions $u \in C([0, T] ; X)$ and $\sigma \in C\left([0, T] ; X^{*}\right)$, equality (5.9) holds if and only if

$$
u(t)=A^{-1} \sigma(t)+\mathcal{R} \sigma(t) \quad \forall t \in[0, T] .
$$

Theorem 20 represents a particular case of two more general results obtained in [30]. Its proof can be obtained by taking $Y=X^{*}$ in Theorems 34 and 35 in [30]. The importance of this theorem arises in the fact that it allows (at least theoretically) to express one of the unknowns of the implicit equation (5.9) in function of the other one. And, for our aim below, it guarantees the existence (and, obviously, the uniqueness) of the operators $\mathcal{S}$ and $\mathcal{R}$ which will be involved in the split problem we consider in this section. Note that the explicit expression $\mathcal{S}$ and $\mathcal{R}$ can be done in several particular cases as shown in [30].

Finally, for each $t \in[0, T]$ we define the set

$$
\Sigma(t)=\left\{\tau \in X^{*}:\langle\tau, v-\widetilde{g}\rangle \geq\langle p(t), v-\widetilde{g}| \forall v \in K\right\} .
$$

Note that the set (5.12) is time-dependent and, in addition, it depends on $K$ and $p$.

With these data we consider the following problem. 
Problem $\mathcal{M}$ Find $x=(u, \sigma) \in C\left([0, T] ; X \times X^{*}\right)$ such that, for all $t \in[0, T]$,

$$
\begin{array}{ll}
u(t) \in K, \quad\langle A u(t)+\mathcal{S} u(t), v-u(t)\rangle \geq\langle p(t), v-u\rangle & \forall v \in K, \\
\sigma(t) \in \Sigma(t), \quad\left\langle\tau-\sigma(t), A^{-1} \sigma(t)+\mathcal{R} \sigma(t)-\widetilde{g}\right\rangle \geq 0 & \forall \tau \in \Sigma(t), \\
\sigma(t)=A u(t)+\int_{0}^{t} B(s, u(s), \sigma(s)) d s . &
\end{array}
$$

It is easy to see that Problem $\mathcal{M}$ is a split variational inequality of the form $\mathcal{M}=$ $\mathcal{M}(P, Q, G, f)$ in which the spaces $X, Y$ and $Z$ are replaced by the spaces $C([0, T] ; X)$, $C\left([0, T] ; X^{*}\right)$ and $C\left([0, T] ; X^{*}\right)$, respectively. Problem $P$ is given by the history-dependent variational inequality (5.13), Problem $Q$ is given by the history-dependent variational inequality (5.14), the operator $G: C([0, T] ; X) \times C\left([0, T] ; X^{*}\right) \rightarrow C\left([0, T] ; X^{*}\right)$ is given by

$$
G(u, \sigma)(t)=A u(t)+\int_{0}^{t} B(s, u(s), \sigma(s)) d s-\sigma(t)
$$

and, finally, $f$ is the zero element of the space $C\left([0, T] ; X^{*}\right)$.

The sets of solutions of these inequalities will be denoted by $S_{P}$ and $S_{Q}$, as usual. Moreover, for any $\varepsilon>0$, the approximating for Problems $P$ and $Q$ are given by

$$
\begin{aligned}
\Omega^{P}(\varepsilon)= & \{u \in C([0, T] ; X): u(t) \in K, \\
& \left.\langle A u(t)+\mathcal{S} u(t)-p(t), v-u(t)| \geq-\varepsilon\|u(t)-v\|_{X} \forall v \in K, t \in[0, T]\right\}, \\
\Omega^{Q}(\varepsilon)= & \left\{\sigma \in C\left([0, T] ; X^{*}\right): \sigma(t) \in \Sigma(t),\right. \\
& \left.\left\langle\tau-\sigma(t), A^{-1} \sigma(t)+\mathcal{R} \sigma(t)-\widetilde{g}\right\rangle \geq-\varepsilon\|\sigma(t)-\tau\|_{X^{*}} \forall \tau \in \Sigma(t), t \in[0, T]\right\} .
\end{aligned}
$$

Using (2.8), it follows that

$$
\begin{aligned}
\Omega^{M}(\varepsilon)= & \left\{x=(u, \sigma): u \in \Omega^{P}(\varepsilon), \sigma \in \Omega^{Q}(\varepsilon),\right. \\
& \left.\left\|A u(t)+\int_{0}^{t} B(s, u(s), \sigma(s)) d s-\sigma(t)\right\|_{X^{*}} \leq \varepsilon \forall t \in[0, T]\right\}
\end{aligned}
$$

and thus the condition (2.9) is, obviously, satisfied.

Our result in the study of Problem $\mathcal{M}$ is the following.

Theorem 21 Assume X is a reflexive Banach space. Then, under the conditions (5.3)-(5.7), the split history-dependent variational inequality $\mathcal{M}$ is well-posed.

Proof We shall use Theorem 14 and, to this end, we start by proving the well-posedness of the history-dependent variational inequalities (5.13) and (5.14).

First, since $X$ is reflexive and $\mathcal{S}$ is a history dependent operator, Theorem 93 in [30] guarantees that, under assumptions (5.3), (5.5), (5.7) problem (5.13) has a unique solution $u \in C([0, T] ; X)$. Assume now that $\left\{u_{n}\right\} \subset C([0, T] ; X)$ is an approximating sequence for (5.13), i.e., $u_{n} \in \Omega^{P}\left(\varepsilon_{n}\right)$ with $0<\varepsilon_{n} \rightarrow 0$. Let $n \in \mathbb{N}$ and $t \in[0, T]$. We use (5.13) with 
$v=u_{n}(t)$ and (5.16) with $v=u(t)$ to see that

$$
\begin{aligned}
& \left\langle A u(t)+\mathcal{S} u(t)-p(t), u_{n}(t)-u(t)\right\rangle \geq 0, \\
& \left\langle A u_{n}(t)+\mathcal{S} u_{n}(t)-p(t), u(t)-u_{n}(t)\right\rangle \geq-\varepsilon_{n}\left\|u_{n}(t)-u(t)\right\|_{X} .
\end{aligned}
$$

We now add the above two inequalities to find that

$$
\begin{aligned}
& \left(A u_{n}(t)-A u(t), u_{n}(t)-u(t)\right)_{X} \\
& \quad \leq \varepsilon_{n}\left\|u_{n}(t)-u(t)\right\|_{X}+\left\langle\mathcal{S} u_{n}(t)-\mathcal{S} u(t), u_{n}(t)-u(t)\right\rangle .
\end{aligned}
$$

On the other hand, since $\mathcal{S}$ is a history-dependent operator, inequality (5.1) implies that

$$
\left\|\mathcal{S} u_{n}(t)-\mathcal{S} u(t)\right\|_{X_{*}} \leq L_{S} \int_{0}^{t}\left\|u_{n}(s)-u(s)\right\|_{X} d s
$$

with $L_{S}>0$. We now combine (5.19), (5.20) and use assumption (5.5) to obtain

$$
\left\|u_{n}(t)-u(t)\right\|_{X} \leq \frac{\varepsilon_{n}}{m_{A}}+\frac{L_{S}}{m_{A}} \int_{0}^{t}\left\|u_{n}(s)-u(s)\right\|_{X} d s .
$$

We now use the Gronwall argument to deduce that

$$
\left\|u_{n}(t)-u(t)\right\|_{X} \leq \frac{\varepsilon_{n}}{m_{A}} e^{\frac{L_{S}}{m_{A}} t}
$$

which, together with (5.2) and the convergence $\varepsilon_{n} \rightarrow 0$, implies that $u_{n} \rightarrow u$ in $C([0, T] ; X)$. Therefore, using Definition 2 we deduce that the variational inequality (5.13) is well-posed. For any $t \in[0, T]$, it is easy to see that the set $\Sigma(t)$ defined by (5.12) satisfies the equality

$$
\Sigma(t)=p(t)+\Sigma_{0},
$$

where $\Sigma_{0}$ is the time-independent set given by

$$
\Sigma_{0}=\left\{\tau \in X^{*}:\langle\tau, v-\widetilde{g}\rangle \geq 0 \text { for all } v \in K\right\} .
$$

Using this property and the regularity (5.7) it is easy to see that a function $\sigma \in C\left([0 ; T] ; X^{*}\right)$ is solution of the history-dependent equation (5.14) if and only if the function $\bar{\sigma}=\sigma-p$ is continuous and satisfies the following auxiliary problem: find $\bar{\sigma}:[0, T] \rightarrow X^{*}$ such that

$$
\begin{gathered}
\bar{\sigma}(t) \in \Sigma_{0}, \quad\left\langle\tau-\bar{\sigma}(t), A^{-1} \bar{\sigma}(t)+A^{-1} p(t)+\mathcal{R}(\bar{\sigma}(t)+p(t))-\widetilde{g}\right) \mid \geq 0 \\
\forall \tau \in \Sigma_{0}, t \in[0, T] .
\end{gathered}
$$

On the other hand, it is easy to see $\Sigma_{0}$ is a convex closed subset of $X^{*}$. Recall also that $A^{-1}: X^{*} \rightarrow X$ is a linear continuous and positively defined operator, $\mathcal{R}: C\left([0, T] ; X^{*}\right) \rightarrow$ $C([0, T] ; X)$ is a history-dependent operator and $X^{*}$ is a reflexive Banach space. Therefore, using again Theorem 93 in [30] and arguments similar to those used in the study to the history-dependent variational inequality (5.13) we deduce that the auxiliary problem 
(5.23) is well-posed. Moreover, using the particular structure (5.21) of the set $\Sigma(t)$, we find that the history-dependent variational inequality (5.14) is well-posed, too. We denote in what follows by $\sigma$ its unique solution.

Let $u \in C([0, T] ; X)$ be the solution of inequality (5.13), $t \in[0, T]$ and, moreover, let

$$
\widetilde{\sigma}(t)=A u(t)+\mathcal{S} u(t)
$$

Then, using Theorem 20(2) and (5.13) we deduce that

$$
u(t)=A^{-1} \widetilde{\sigma}(t)+\mathcal{R} \widetilde{\sigma}(t)
$$

and, moreover,

$$
\langle\widetilde{\sigma}(t), v-u(t)\rangle \geq\langle p(t), v-u(t)\rangle \quad \forall v \in K
$$

We now use assumptions (5.4)(a) and (b) and test successively in (5.26) with $v=2 u(t)-\tilde{g}$ and $v=\widetilde{g}$. As a result we find that

$$
\langle\widetilde{\sigma}(t), u(t)-\widetilde{g}\rangle=\langle p(t), u(t)-\widetilde{g}\rangle
$$

We now combine inequality (5.26) and equality (5.27) to see that

$$
\langle\widetilde{\sigma}(t), v-\widetilde{g}\rangle \geq\langle p(t), v-\widetilde{g}\rangle \quad \forall v \in K,
$$

which shows that

$$
\tilde{\sigma} \in \Sigma(t)
$$

Let $\tau \in X^{*}$. We use Eqs. (5.25) and (5.27) to see that

$$
\begin{aligned}
\left\langle\tau-\tilde{\sigma}(t), A^{-1} \tilde{\sigma}(t)+\mathcal{R} \widetilde{\sigma}(t)-\tilde{g}\right\rangle & =\langle\tau-\widetilde{\sigma}(t), u(t)-\widetilde{g}\rangle \\
& =\langle\tau, u(t)-\widetilde{g}\rangle-\langle p(t), u(t)-\widetilde{g}\rangle
\end{aligned}
$$

and, since $u(t) \in K$, definition (5.12) yields

$$
\left\langle\tau-\widetilde{\sigma}(t), A^{-1} \widetilde{\sigma}(t)+\mathcal{R} \widetilde{\sigma}(t)-\widetilde{g}\right| \geq 0 \quad \forall \tau \in \Sigma(t) .
$$

We now combine Eqs. (5.28) and (5.29) to deduce that the function $\widetilde{\sigma}$, which clearly belongs to $C\left([0, T], X^{*}\right)$, is a solution of the history-dependent variational inequality (5.14). On the other hand, we know that this inequality has a unique solution, denoted by $\sigma$. It follows from this that $\widetilde{\sigma}=\sigma$ and, therefore, using (5.24) we find that $\sigma=A u(t)+\mathcal{S} u(t)$. Finally, using Theorem 201 ) we deduce that (5.15) holds.

To conclude, we proved that inequalities (5.13) and (5.14) are well-posed and their solutions are such that (5.15) holds. We are now in a position to use Theorem 14 1) to conclude that the split history-dependent variational inequality $\mathcal{M}$ is well-posed, which completes the proof. 
We end this section with the remark that split history-dependent variational inequalities of the form (5.13)-(5.15) arise in the study of quasistatic frictionless contact problems with unilateral constraints for viscoelastic or rate-type viscoplastic materials. There, $u$ represents the displacement field and $\sigma$ is related to the stress field. The operators $A$ and $B$ describe the properties of the material, the set $K$ is determined by the unilateral contact conditions used in the model and $p$ is related to the external forces and tractions acting on the deformable body. Inequality (5.13) represents the variational formulation of the contact problem in terms of displacements, the so-called primal variational formulation. Inequality (5.14) represents the variational formulation in terms of stress of the contact problem, the so-called dual variational formulation. Finally, Eq. (5.15) is related to the constitutive law of the material. More details, comments and physical interpretation on such kind of problems can be found in $[29,30]$. Here we restrict ourselves to the following one-dimensional example.

Example 22 Consider the following initial and boundary problem.

Problem $P_{\text {Sig }}$ Find a displacement field $u:[0,1] \times[0, T] \rightarrow \mathbb{R}$ and a stress field $\widetilde{\sigma}:[0,1] \times$ $[0, T] \rightarrow \mathbb{R}$ such that

$$
\begin{aligned}
& \widetilde{\sigma}(x, t)=E \frac{\partial u}{\partial t}(x, t)+\eta \int_{0}^{t} e^{-(t-s)} \frac{\partial u}{\partial x}(x, s) d s \quad \forall(x, t) \in(0,1) \times(0, T), \\
& \frac{\partial \widetilde{\sigma}(x, t)}{\partial x}+p(x, t)=0 \quad \forall(x, t) \in(0,1) \times(0, T), \\
& u(0, t)=0 \quad \forall t \in(0, T), \\
& u(1, t) \leq 0, \widetilde{\sigma}(1, t) \leq 0, \quad \widetilde{\sigma}(1, t) u(1, t)=0 \quad \forall t \in(0, T) .
\end{aligned}
$$

Problem $P_{\text {Sig }}$ is a Signorini-type one-dimensional problem; see [30] for details. It describes the equilibrium of a viscoelastic rod which occupies the interval $[0,1]$ on the $O x$ axis, is fixed at $x=0$ and is in contact at $x=1$ with an obstacle. The interval of time of interest is $[0, T]$ with $T>0$. Equation (5.30) represents the constitutive law of the material in which $E$ is the Young modulus and $\eta$ is a relaxation coefficient. Equation (5.31) is the equation of equilibrium in which $p$ denotes the density of body forces and (5.32) represents the displacement boundary condition. Finally, conditions (5.33) represent the Signorini conditions which model the contact with a rigid obstacle.

For the variational analysis of Problem $P_{\text {Sig }}$ we use the space $X$ given by

$$
X=\left\{v \in H^{1}(0,1): v(0)=0\right\}
$$

equipped with the inner product

$$
(u, v)_{X}=\int_{0}^{1} \frac{d u}{d x} \cdot \frac{d v}{d x} d x
$$

and the associated norm $\|\cdot\|_{X}$. We denote by $X^{*}$ the dual of $X$ and, as usual, we use $\langle\cdot, \cdot\rangle$ for the dualty pairing mapping. Assume that

$$
E>0, \quad \eta>0, \quad p \in C\left([0, T] ; L^{2}(0,1)\right) \subset C\left([0, T] ; X^{*}\right) .
$$


Then, we define the set $K$ and operators $A: X \rightarrow X^{*}, B:[0, T] \times X \times X^{*} \rightarrow X^{*}$ by equalities

$$
\begin{aligned}
& K=\{v \in X: v(1) \leq 0\} \\
& \langle A u, v\rangle=E \int_{0}^{1} \frac{d u}{d x} \cdot \frac{d v}{d x} d x \quad \forall u, v \in X, \\
& \langle B(t, u, \sigma), v\rangle=\eta \int_{0}^{1} e^{-(t-s)} \frac{d u}{d x} \cdot \frac{d v}{d x} d x \quad \forall t \in[0, T], u, v \in X, \sigma \in X^{*} .
\end{aligned}
$$

Note that in this case conditions (5.3)-(5.7) are satisfied with $\widetilde{g}=0_{X}, m_{A}=E$ and $L_{B}=\eta$. Next, we use the Riesz representation theorem to introduce the function $\sigma:[0, T] \rightarrow X^{*}$ related to the stress field $\widetilde{\sigma}$ by equality

$$
\langle\sigma(t), v\rangle=\int_{0}^{1} \tilde{\sigma}(t) \frac{d v}{d x} d x \quad \forall v \in X, t \in[0, T]
$$

Note that (5.39) and (5.30) imply that (5.9) holds with $A$ and $B$ defined above. Therefore, we are in a position to use Theorem 20 to find two history-dependent operators $\mathcal{S}: C([0, T] ; X) \rightarrow C\left([0, T] ; X^{*}\right)$ and $\mathcal{R}: C\left([0, T] ; X^{*}\right) \rightarrow C([0, T] ; X)$ such that $(5.10)$ and (5.11) hold. Note that in this particular case we can give the following explicit expression for the operator $\mathcal{S}$ :

$$
\langle\mathcal{S} u(t), v\rangle=\eta \int_{0}^{t} e^{-(t-s)}\left(\int_{0}^{1} \frac{\partial u}{\partial x}(x, s) \frac{d v}{d x} d x\right) d s
$$

for all $u \in C([0, T] ; X), t \in[0, T], v \in X$.

Using now standard arguments and (5.31)-(5.33), it is easy to see that

$$
u(t) \in K, \quad \int_{0}^{1} \tilde{\sigma}(t)\left(\frac{\partial u}{\partial x}(x, t)-\frac{d v}{d x}\right) d x \geq \int_{0}^{1} p(t)(v-u(t)) d x \quad \forall v \in K, t \in[0, T]
$$

and, therefore, (5.39) yields

$$
u(t) \in K, \quad\langle\sigma(t), v-u(t)\rangle \geq\langle p(t), v-u(t)\rangle \quad \forall v \in K, t \in[0, T] .
$$

We now combine (5.41) and (5.10) to obtain the following variational formulation of Problem $P_{\text {Sig }}$ in terms of displacements.

Problem $P$ Find $u:[0, T] \rightarrow X$ such that, for all $t \in[0, T]$,

$$
u(t) \in K, \quad\langle A u(t)+\mathcal{S} u(t), v-u(t)\rangle \geq\langle p(t), v-u\rangle \quad \forall v \in K .
$$

Next, for each $t \in[0, T]$, we define the set

$$
\Sigma(t)=\left\{\tau \in X^{*}:\langle\tau, v\rangle \geq\langle p(t), v\rangle \forall v \in K\right\}
$$

Then, the variational formulation of Problem $P_{\text {Sig }}$ in terms of stress, obtained by testing in (5.41) with $v=2 u(t)$ and $v=0_{X}$ and using (5.11), is as follows. 
Problem $Q$ Find $\sigma:[0, T] \rightarrow X^{*}$ such that, for all $t \in[0, T]$,

$$
\sigma(t) \in \Sigma(t), \quad\left\langle\tau-\sigma(t), A^{-1} \sigma(t)+\mathcal{R} \sigma(t)\right| \geq 0 \quad \forall \tau \in \Sigma(t)
$$

Problems $P$ and $Q$ represent the primal and dual variational formulations of the Signorini contact problem $P_{\mathrm{Sig}}$, respectively. Recall that the solutions of these problems are related by the equality (5.9). Therefore, gathering these ingredients, it is natural to consider the following split history-dependent variational inequality.

Problem $\mathcal{M}$ Find $x=(u, \sigma)$ such that $u$ is a solution of (5.42), $\sigma$ is a solution of (5.44) and (5.9) holds.

Then, it is easy to see that, under the assumption (5.36)-(5.38), we can apply Theorem 21 in order to find that Problem $\mathcal{M}$ is well-posed.

\section{Acknowledgements}

Not applicable.

\section{Funding}

This research was supported by the National Natural Science Foundation of China (11771067), the Applied Basic Project of Sichuan Province (2019YJ0204), the Fundamental Research Funds for the Central Universities (ZYGX2019J095), and the Scientific Research Project of Chongqing University of Education (KY201923C). This project also received funding from the European Union's Horizon 2020 Research and Innovation Programme under the Marie Sklodowska-Curie Grant Agreement No 823731 CONMECH.

\section{Availability of data and materials}

Not applicable.

\section{Competing interests}

The authors declare that they have no competing interests.

\section{Authors' contributions}

All authors contributed equally and significantly in writing this paper. All authors read and approved the final manuscript.

\section{Author details}

${ }^{1}$ School of Mathematics and Information Engineering, Chongqing University of Education, Chongqing, P.R. China.

${ }^{2}$ School of Mathematical Sciences, University of Electronic Science and Technology of China, Chengdu, P.R. China.

${ }^{3}$ Laboratoire de Mathématiques et Physique, University of Perpignan Via Domitia, Perpignan, France.

\section{Publisher's Note}

Springer Nature remains neutral with regard to jurisdictional claims in published maps and institutional affiliations.

Received: 21 April 2020 Accepted: 25 May 2020 Published online: 03 June 2020

\section{References}

1. Baiocchi, C., Capelo, A.: Variational and Quasivariational Inequalities: Applications to Free-Boundary Problems. Wiley, Chichester (1984)

2. Cai, D.L., Sofonea, M., Xiao, Y.B.: Convergence results for elliptic variational-hemivariational inequalities. Adv. Nonlinear Anal. 10, 2-23 (2021)

3. Cai, D.L., Sofonea, M., Xiao, Y.B.: Tykhonov well-posedness of a mixed variational problem. Submitted

4. Capatina, A.: Variational Inequalities and Frictional Contact Problems. Advances in Mechanics and Mathematics, vol. 31, pp. 31-131. Springer, New York (2014)

5. Censor, Y., Bortfeld, T., Martin, B., Trofimov, A.: A unified approach for inversion problems in intensity-modulated radiation therapy. Phys. Med. Biol. 51, 2353-2365 (2006)

6. Censor, Y., Gibali, A., Reich, S.: Algorithms for the split variational inequality problem. Numer. Algorithms 59, 301-323 (2012)

7. Clarke, F.H.: Optimization and Nonsmooth Analysis. Interscience, New York (1983)

8. Duvaut, G., Lions, J.-L.: Inequalities in Mechanics and Physics. Springer, Berlin (1976)

9. Ekeland, I., Temam, R.: Convex Analysis and Variational Problems. SIAM, Philadelphia (1976)

10. Glowinski, R., Lions, J.-L., Trémolières, R.: Numerical Analysis of Variational Inequalities. Elsevier, Amsterdam (1981)

11. Goeleven, D., Mentagui, D.: Well-posed hemivariational inequalities. Numer. Funct. Anal. Optim. 16, 909-921 (1995)

12. He, H., Ling, C., Xu, H.K.: A relaxed projection method for split variational inequalities. J. Optim. Theory Appl. 166 $213-233(2015)$ 
13. Hu, R., Sofonea, M., Xiao, Y.B.: Tykhonov triples and convergence results for hemivariational inequalities. Submitted

14. Hu, R., Sofonea, M., Xiao, Y.B.: A Tykhonov-type well-posedness concept for elliptic hemivariational inequalities. Z. Angew. Math. Phys. (2020, in press)

15. Hu, R., Xiao, Y.B., Huang, N.J., Wang, X.: Equivalence results of well-posedness for split variational-hemivariational inequalities. J. Nonlinear Convex Anal. 20,447-459 (2019)

16. Kinderlehrer, D., Stampacchia, G.: An Introduction to Variational Inequalities and Their Applications. Academic Press, New York (1980)

17. Kinderlehrer, D., Stampacchia, G.: An Introduction to Variational Inequalities and Their Applications. Classics in Applied Mathematics, vol. 31, pp. 222-274. SIAM, Philadelphia (2000)

18. Kurdila, A.J., Zabarankin, M.: Convex Functional Analysis. Birkhäuser, Basel (2005)

19. Lucchetti, R., Patrone, F.: A characterization of Tychonov well-posedness for minimum problems with applications to variational inequalities. Numer. Funct. Anal. Optim. 3, 461-476 (1981)

20. Luchetti, R., Patrone, F.: Some properties of "well-posed" variational inequalities governed by linear operators. Numer. Funct. Anal. Optim. 5, 349-361 (1983)

21. Migórski, S., Ochal, A., Sofonea, M.: Nonlinear Inclusions and Hemivariational Inequalities: Models and Analysis of Contact Problems. Advances in Mechanics and Mathematics, vol. 26, pp. 95-169. Springer, New York (2013)

22. Migórski, S., Ochal, A., Sofonea, M.: A class of variational-hemivariational inequalities in reflexive Banach spaces. J. Elast. 127, 151-178 (2017)

23. Moudafi, A.: Split monotone variational inclusions. J. Optim. Theory Appl. 150, 275-283 (2011)

24. Naniewicz, Z., Panagiotopoulos, P.D.: Mathematical Theory of Hemivariational Inequalities and Applications. CRC Press, New York (1995)

25. Panagiotopoulos, P.D.: Inequality Problems in Mechanics and Applications. Birkhäuser, Boston (1985)

26. Panagiotopoulos, P.D.: Hemivariational Inequalities. Springer, Berlin (1993)

27. Shu, Q.Y., Hu, R., Xiao, Y.B.: Metric characterizations for well-posedness of split hemivariational inequalities. J. Inequal. Appl. (2018). https://doi.org/10.1186/s13660-018-1761-4

28. Sofonea, M., Matei, A.: Variational Inequalities with Applications: A Study of Antiplane Frictional Contact Problems. Springer, New York (2009)

29. Sofonea, M., Matei, A.: Mathematical Models in Contact Mechanics. Cambridge University Press, London (2012)

30. Sofonea, M., Migórski, S.: Variational-Hemivariational Inequalities with Applications. Chapman \& Hall/CRC Press, London (2018)

31. Sofonea, M., Xiao, Y.B.: Boundary optimal control of a nonsmooth frictionless contact problem. Comput. Math. Appl. 78, 152-165 (2019)

32. Sofonea, M., Xiao, Y.B.: On the well-posedness concept in the sense of Tykhonov. J. Optim. Theory Appl. 183, 139-157 (2019)

33. Sofonea, M., Xiao, Y.B.: Tykhonov well-posedness of a viscoplastic contact problem. Evol. Equ. Control Theory (2020, in press)

34. Sofonea, M., Xiao, Y.B., Couderc, M.: Optimization problems for elastic contact models with unilateral constraints Z. Angew. Math. Phys. (2019). https://doi.org/10.1007/s00033-018-1046-2

35. Sofonea, M., Xiao, Y.B., Couderc, M.: Optimization problems for a viscoelastic frictional contact problem with unilateral constraints. Nonlinear Anal., Real World Appl. 50, 86-103 (2019)

36. Tykhonov, A.N.: On the stability of functional optimization problems. USSR Comput. Math. Math. Phys. 6, 631-634 (1966)

37. Xiao, Y.B., Huang, N.J., Wong, M.M.: Well-posedness of hemivariational inequalities and inclusion problems. Taiwan. J. Math. 15, 1261-1276 (2011)

38. Xiao, Y.B., Sofonea, M.: On the optimal control of variational-hemivariational inequalities. J. Math. Anal. Appl. 475, 364-384 (2019)

39. Xiao, Y.B., Sofonea, M.: Generalized penalty method for elliptic variational-hemivariational inequalities. Appl. Math. Optim. (2019). https://doi.org/10.1007/s00245-019-09563-4

40. Xiao, Y.B., Sofonea, M.: Tykhonov triples, well-posedness and convergence results. Submitted

41. Zeidler, E.: Nonlinear Functional Analysis and Applications II A/B. Springer, New York (1990)

42. Zhou, Z.A., Chen, W., Yang, X.M.: Scalarizations and optimality of constrained set-valued optimization using improvement sets and image space analysis. J. Optim. Theory Appl. 183, 944-962 (2019)

\section{Submit your manuscript to a SpringerOpen ${ }^{\circ}$ journal and benefit from:}

- Convenient online submission

- Rigorous peer review

- Open access: articles freely available online

- High visibility within the field

Retaining the copyright to your article

Submit your next manuscript at $\boldsymbol{s p r i n g e r o p e n . c o m ~}$ 